\title{
Flow-induced forces arising during the impact of two circular cylinders
}

\author{
N. B A MPALAS AND J. M. R. GRAHAM† \\ Department of Aeronautics, Imperial College, London, SW7 2AZ, UK
}

(Received 03 July 2007 and in revised form 19 March 2008)

This paper presents numerical simulations of two-dimensional incompressible flow around two circular cylinders in relative motion, which may result in impact. Viscous flow computations are carried out using a streamfunction-vorticity method for two equal-diameter cylinders undergoing a two-dimensional impact in otherwise stationary fluid and for cases of similar impact of two cylinders in a steady incident flow. These results are supported by potential flow calculations carried out using a Möbius conformal transformation and infinite arrays of image singularities. The inviscid flow results are compared with other published work and show that the inviscid forces induced on the cylinders have an inverse square root singularity with respect to the time to impact. All impacts considered in this paper result from steady motion of the cylinders along the line joining their centres.

\section{Introduction}

Deepwater recovery of oil and gas from sub-sea reservoirs frequently involves the deployment of very long, flexible riser pipes between the surface-production platform and the seabed. These pipes are deployed in groups and subject to currents which cause motion due to vortex-induced vibration (VIV) (Vandiver 1993) and wake buffeting (Bokaian \& Geola 1984). As a result of these motions adjacent risers sometimes impact (Sagatun et al. 1999). Current industry practice is to design riser separations sufficient to avoid this if possible, which increases platform costs. The issue of impact damage is not clear, and impact coatings to protect risers are also considered. A related problem, which has attracted a considerable amount of research, is that of calculating the flow field around two fixed cylinders in close proximity in a uniform incident flow. Zdravkovich (2003) has described a large number of such cases, which are highly relevant to exterior flow around free pipeline spans and to more complex flows which arise in heat transfer equipment. Previous investigation of the forces arising when two bodies impact one another in a fluid has mainly focused on spheres and circular cylinders and is often based on potential flow analysis. The potential flow field of two cylinders in proximity in a uniform flow, or equivalently of a cylinder close to a plane wall bounding a uniform flow, is usually computed by setting up the dipole image system which satisfies the normal-velocity boundary condition on the solid surfaces. This results in an infinite series of dipoles within the bodies. A first such attempt to calculate the inviscid force during the impact of two circular cylinders immersed in inviscid fluid was made by Hicks (1879), who also investigated the similar problem of two spheres moving in a fluid (Hicks 1880). Dalton \& Helfinstine (1971) developed the

$\dagger$ Email address for correspondence: m.graham@imperial.ac.uk 

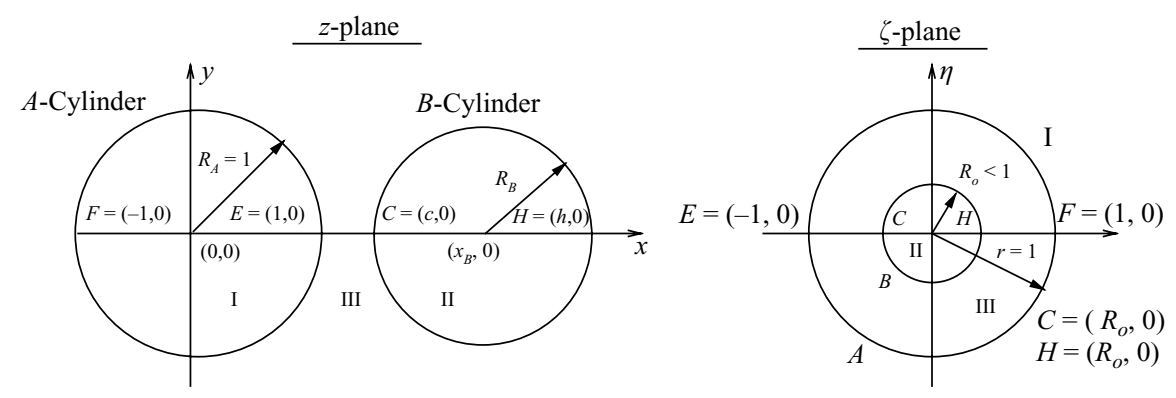

Figure 1. The physical and the transformed planes.

image source method involving an infinite array of images, to model two-dimensional potential flow around a group of circular cylinders in an unsteady incident flow; this problem is relevant to forces induced by waves and motion of elements of offshore oil platforms. They computed lift, drag and inertia coefficients for a range of fixed geometries and separations including cases of cylinders in contact. Small separations were shown to have a large effect on the force coefficients. Wang (2004) used a different two-dimensional method to evaluate the inviscid force developed between two cylinders. Using a Möbius conformal transformation and Fourier series he derived general formulae for the unsteady problem of two circular cylinders expanding and translating arbitrarily. Crowdy (2006) considered the steady irrotational flow problem of a uniform stream around multiple circular cylinders of zero circulation. In Landweber Chwang \& Guo (1991) and ? an integral-equation procedure was used to investigate the problem of an ice mass approaching an offshore structure moving towards a central impact. This problem was simplified to the case of a moving cylinder approaching a stationary one until impact.

In the present work various cases are considered of the unsteady problem of the impact of two equal-size circular cylinders. In the first part, the fluid is considered inviscid, and the behaviour of the potential flow induced force is investigated and compared with other inviscid studies. In the second part of the paper the same problems are considered for viscous flow by solving the streamfunction-vorticity form of the Navier-Stokes equations.

The main aim of the paper is to determine the behaviour of the force between the cylinders right up to the moment of impact. Although the 'perfect' normal twodimensional impact of two smooth circular cylinders never occurs exactly, the force which arises provides an upper limit to the hydrodynamic forces which are generated by more general impacts. The inviscid analysis done first, allows the analytical behaviour of the force to be investigated, which in turn helps in the evaluation of the viscous forces.

An important issue in multi-body flows is the volume flux passing between the bodies, and the (inviscid) evaluation of the difference $\Delta \Psi$ in the surface values of the streamfunction on the bodies is relevant for the viscous streamfunction-vorticity flow computations.

\section{Potential flow theory}

\subsection{Möbius conformal transformation}

The two-dimensional potential flow field around two cylinders in the axis system shown in figure 1 is considered. The flow may be due to motion of the fluid at infinity 
or relative motion of the cylinders or both. The Möbius conformal transformation

$$
\zeta=\frac{z-\lambda}{\lambda z-1}
$$

is applied to transform this flow field into that between two concentric cylinders, where $z=x+\mathrm{i} y$ in the physical plane and $\zeta=\xi+\mathrm{i} \eta$ in the transformed plane (see figure 1 and, e.g. Saff \& Snider 2003). In (2.1)

$$
\lambda=\frac{1+c h+\sqrt{\left(c^{2}-1\right)\left(h^{2}-1\right)}}{c+h} .
$$

In the transformed plane, the centres of both circles are located at the origin. The outer cylinder has a radius equal to one, while the radius $R_{o}$ of the inner circle is

$$
R_{o}=\frac{c h-1-\sqrt{\left(c^{2}-1\right)\left(h^{2}-1\right)}}{h-c} .
$$

In general cases since the cylinders may have circulation it is convenient to work with the streamfunction $\Psi$ which is single-valued, and some basic results for $\Psi$ are now derived.

\subsection{Streamfunction for a point vortex in the presence of two circular cylinders}

We start by examining the flow field around two stationary cylinders in the presence of a single vortex at $z_{o}=x_{o}+\mathrm{i} y_{o}$. This fundamental case can also provide as a dipole the main component of the analysis in the transformed plane in which a free stream is present as well as the method of calculating $\Delta \Psi$ in the viscous streamfunction-vorticity computations. It is necessary to consider various possibilities for the circulation around the cylinders. The case in which the whole flow field has zero circulation made up of zero circulation around one of the cylinders and a 'balancing' circulation equal and opposite to that of the point vortex in the flow field around the other is considered first. The case of a point vortex in the flow field and zero circulation around both cylinders may be evaluated from this. The case of arbitrary circulation around the cylinders is considered in the next section.

The point in the transformed plane into which the vortex transforms under the Möbius transformation is denoted by $\zeta_{o}=\xi_{o}+\mathrm{i} \eta_{o}=R \mathrm{e}^{\mathrm{i} \phi_{o}}$. The complex potential due to a point vortex of circulation $\Gamma$ placed in isolation at this point is

$$
w(\zeta)=\mathrm{i} \kappa \ln \left(\zeta-\zeta_{o}\right),
$$

where $\kappa=\Gamma /(2 \pi)$, is the strength of the vortex. If we transform this potential back to the $z$-plane we obtain corresponding to the single vortex in the $\zeta$ plane

$$
w(z)=\mathrm{i} \kappa \ln \left(z-z_{o}\right)-\mathrm{i} \kappa \ln \left(z-\frac{1}{\lambda}\right)+\text { constant, }
$$

the potential for a vortex of circulation $\Gamma$ at $z_{o}=\left(\zeta_{o}-\lambda\right) /\left(\lambda \zeta_{o}-1\right)$ together with $-\Gamma$ at $z=1 / \lambda$ inside cylinder $A$. Hence the case of a single vortex in the flow together with opposite circulation on one cylinder is the simplest to consider first. Using (2.2), an appropriate image system to satisfy the no-penetration boundary condition on both circular boundaries can now be constructed. This consists of an infinite array of image vortices. Each image in one cylinder of a given vortex generates a further image in the outer cylinder and so on. The full image system, presented in tables 1 and 2 , shows consistently that the circulation around the inner cylinder is zero and around the outer one is equal to $\Gamma$. (The direction of the outer 


\begin{tabular}{|c|c|c|c|c|}
\hline & $\begin{array}{l}\text { Strength, } \\
\text { region I }\end{array}$ & $\begin{array}{l}\text { Radial coordinate, } \\
\text { region I }\end{array}$ & $\begin{array}{l}\text { Strength, } \\
\text { region II }\end{array}$ & $\begin{array}{l}\text { Radial coordinate } \\
\text { region II }\end{array}$ \\
\hline$i=1$ & $-\kappa$ & $1 / \xi_{o}$ & $-\kappa$ & $R_{o}^{2} / \xi_{o}$ \\
\hline$i=2$ & $\kappa$ & $\xi_{o} / R_{o}^{2}$ & $\kappa$ & $R_{o}^{2} \xi_{o}$ \\
\hline$i=3$ & $-\kappa$ & $1 /\left(\xi_{o} R_{o}^{2}\right)$ & $-\kappa$ & $R_{o}^{4} / \xi_{o}$ \\
\hline$i=4$ & $\kappa$ & $\xi_{o} / R_{o}^{4}$ & $\kappa$ & $\xi_{o} R_{o}^{4}$ \\
\hline$i=5$ & $-\kappa$ & $1 /\left(\xi_{o} R_{o}^{4}\right)$ & $-\kappa$ & $R_{o}^{6} / \xi_{o}$ \\
\hline : & $\vdots$ & $\vdots$ & $\vdots$ & $\vdots$ \\
\hline
\end{tabular}

TABLE 1. Position and strength of the point vortices, generated using the method of images in the $\zeta$-plane.

\begin{tabular}{ccccc}
\hline Index & $\begin{array}{c}\text { Strength, } \\
\text { region I }\end{array}$ & $\begin{array}{c}\text { Radial coordinate, } \\
\text { region I }\end{array}$ & $\begin{array}{c}\text { Strength, } \\
\text { region II }\end{array}$ & $\begin{array}{c}\text { Radial coordinate, } \\
\text { region II }\end{array}$ \\
Odd & $-\kappa$ & $1 /\left(\xi_{o} R_{o}^{i-1}\right)$ & $-\kappa$ & $R_{o}^{i+1} / \xi_{o}$ \\
Even & $\kappa$ & $\xi_{o} / R_{o}^{i}$ & $\kappa$ & $\xi_{o} R_{o}^{i}$
\end{tabular}

TABLE 2. Position and strength of the arbitrary $i$ th image vortex in the $\zeta$-plane, where $(i=1,2, \ldots \infty)$.

cylinder reverses under the transformation, and the image system within the inner cylinder is constructed from pairs of vortices of equal and opposite circulation.) The advantage of using the Möbius transformation is that the whole system of images in the $\zeta$-plane is co-linear. Using De Moivre's theorem and a standard expansion for the logarithmic function, the imaginary part of the complex potential (at $\zeta=r \mathrm{e}^{\mathrm{i} \phi}$ ) generated by the point vortex, at $R \mathrm{e}^{\mathrm{i} \phi_{o}}$, in the presence of the pair of cylinders is given for this case by the following equations:

$$
\left.\begin{array}{ll}
\Psi=\kappa \ln R-\kappa \sum_{n=1}^{\infty}\left[\left(r R^{-1}\right)^{n} n^{-1} \cos n \theta\right] & r / R_{o} \leqslant 1, \\
\Psi=\kappa \ln r-\kappa \sum_{n=1}^{\infty}\left[\left(R r^{-1}\right)^{n} n^{-1} \cos n \theta\right] & r / R_{o}>1,
\end{array}\right\}
$$

where $\theta=\phi-\phi_{o}$. Using the coordinates and strengths of the image vortices given in table 2, and manipulating the infinite sums, cancellation of equal and opposite terms gives

$$
\begin{aligned}
& \Psi=\kappa \ln R-\kappa \sum_{n=1}^{\infty}\left[\left(r R^{-1}\right)^{n} n^{-1} \cos n \theta\right]+\kappa \sum_{n=1}^{\infty}\left[(r R) n^{-1} \cos n \theta\right] \\
& +\kappa \sum_{n=1}^{\infty}\left\{n^{-1} \cos n \theta\left(R^{n}-R^{-n}\right)\left(r^{n}-r^{-n}\right)\left[R_{o}^{2 n}\left(1-R_{o}^{2 n}\right)^{-1}\right]\right\} \quad r / R_{o} \leqslant 1, \\
& \Psi=\kappa \ln r-\kappa \sum_{n=1}^{\infty}\left[\left(R r^{-1}\right)^{n} n^{-1} \cos n \theta\right]+\kappa \sum_{n=1}^{\infty}\left[(r R) n^{-1} \cos n \theta\right] \\
& +\kappa \sum_{n=1}^{\infty}\left\{n^{-1} \cos n \theta\left(R^{n}-R^{-n}\right)\left(r^{n}-r^{-n}\right)\left[R_{o}^{2 n}\left(1-R_{o}^{2 n}\right)^{-1}\right]\right\} \quad r / R_{o}>1,
\end{aligned}
$$




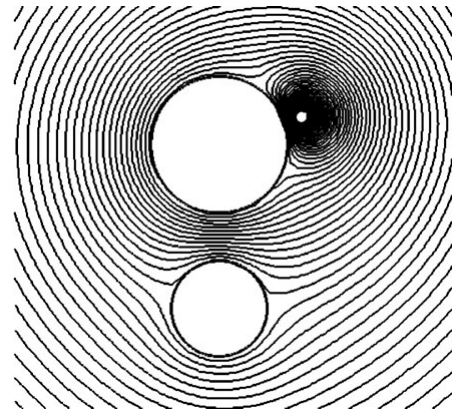

(a)

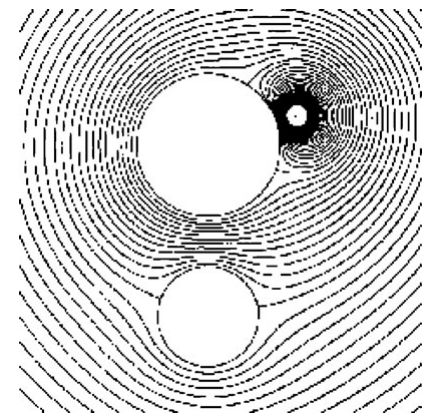

(b)

FIGURE 2. Potential flow streamlines for a point vortex $(a)$ given by $(2.5)$ and $(b)$ calculated by Lee (2000) (published with permission).

The first three terms of both $(2.4 a)$ and $(2.4 b)$ sum to the same expression for $R_{o} \leqslant r \leqslant 1$ :

$$
\begin{aligned}
\Psi(r, \phi)=\kappa \ln \sqrt{R^{2}-2 r R \cos \theta+r^{2}}-\kappa \ln \sqrt{1-2 r R \cos \theta r^{2} R^{2}} \\
+\kappa \sum_{n=1}^{\infty}\left[\frac{\cos n \theta}{n}\left(R^{n}-\frac{1}{R^{n}}\right)\left(r^{n}-\frac{1}{r^{n}}\right)\left(\frac{R_{o}^{2 n}}{1-R_{o}^{2 n}}\right)\right] .
\end{aligned}
$$

Using (2.5), the streamfunction value at the boundaries, $r=1$ and $r=R_{o}$, can also be evaluated. This gives the difference $\Delta \Psi$ between the streamfunction value on the two cylinders; $\Delta \Psi$ is the flux between the cylinders due to the flow and is required for the boundary condition on $\Psi$ in a streamfunction-vorticity computation. Quite often computations of flow fields, which are more than doubly connected, assume a value for this rather than evaluating it independently. From (2.5)

$$
\Psi(1, \phi)=0 \quad \text { and } \quad \Psi\left(R_{o}, \phi\right)=\kappa \ln R .
$$

Therefore, the streamfunction difference between the two cylinders for this case is

$$
\Delta \Psi=\Psi(1)-\Psi\left(R_{o}\right)=-\kappa \ln R,
$$

where $R$ is the radial coordinate of the vortex position in the transformed plane.

Equation (2.6) shows that the flux between two cylinders, one with zero circulation and the other with circulation equal and opposite to the point vortex, together with the point vortex in the flow field depends only on the strength of the vortex and its position in the $\zeta$-plane. Omitting the circulation $(-\Gamma)$ around cylinder $A$ leads to a term $-i \kappa \ln \left(\zeta-\lambda^{-1}\right)$ in $w(\zeta)$ representing the net circulation $\Gamma$ at infinity of the whole flow field (with $\zeta=\lambda^{-1}$ corresponding to $z=\infty$ ). This circulation may be treated similarly as a point vortex with a similar array of images to table 1 in the $\zeta$-plane.

Thus, $\Delta \Psi$ between two cylinders in the $z$-plane, due to the presence of a point vortex of circulation $\Gamma$, with neither of the two cylinders having circulation, is

$$
\Delta \Psi=-\kappa \ln (\lambda R) .
$$

The streamline contours generated by the present method for this case with a point vortex at a point $(-0.4,1.2)$ of the physical plane and the ratio of cylinder radii $R_{B} / R_{A}=0.7$, are compared in figure 2 with those given by Lee (2000). 
2.3. Streamfunction for arbitrary circulation around two circular cylinders (in the absence of point vortices in the flow field)

The flow induced by arbitrary circulation around the $A$ - and $B$-cylinder (see figure 1), $\Gamma_{A}$ and $\Gamma_{B}$ respectively, in the absence of point vortices in the flow field is considered next. This case can be constructed using the result of the previous section, by a combination of two point vortices in the $\zeta$-plane: a point vortex of circulation $\Gamma_{B}$ at the centre of the concentric circles and a point vortex of circulation $-\left(\Gamma_{A}+\Gamma_{B}\right)$ at the singular point $\left(\lambda^{-1}, 0\right)$. Using $(2.7)$, the streamfunction difference for this case is

$$
\Delta \Psi=-\kappa_{A} \ln \lambda-\kappa_{B} \ln \left(\lambda R_{o}\right) .
$$

Any case of a flow containing point vortices can now been obtained from a linear sum of the above results.

\subsection{Streamfunction for a uniform stream incident on two circular cylinders}

We consider the flow field due to a free stream around two cylinders, neither of which have any circulation. Consider two cylinders on the horizontal axis of the physical plane, subject to a free stream at an angle $\alpha$ relative to this axis. Then, the complex potential due to the free stream is

$$
w(z)=U_{\infty} z \mathrm{e}^{-\mathrm{i} \alpha} .
$$

Under the Möbius transformation, the free stream complex potential transforms to

$$
w(\zeta)=-U_{\infty}\left(\frac{1-\lambda^{-2}}{\zeta-\lambda^{-1}}\right) \mathrm{e}^{-\mathrm{i} \alpha}+\text { constant },
$$

which represents the complex potential of a dipole of strength $\mu=-U_{\infty}\left(1-\lambda^{-2}\right)$ at the singular point $\left(\lambda^{-1}, 0\right)$ of the transformed plane. The direction of the dipole's axis depends upon the incidence angle $\alpha$ of the free stream.

The general case can be derived by considering the two basic cases of $\alpha=90^{\circ}$ (cylinders side-by-side with respect to the free stream) and $\alpha=0^{\circ}$ (tandem arrangement).

The first of these $\left(\alpha=90^{\circ}\right)$ is equivalent to a flow due to a source-sink dipole at the point $\left(\lambda^{-1}, 0\right)$ in the transformed plane with its axis at right angles to the real axis or equivalently a doublet vortex (Milne-Thomson 1968), with its axis parallel to the real axis. The streamfunction for this may therefore be obtained from the analysis for a single vortex in $\S 2.2$.

Consider two vortices of equal and opposite strength, placed in the transformed plane, at $(a+\epsilon, 0)$ and $(a-\epsilon, 0)$ respectively, where $a=\lambda^{-1}$ and $0<\epsilon \ll 1$. The vortex dipole streamfunction is obtained in the limit $\kappa \rightarrow \infty$ and $\epsilon \rightarrow 0$ with $\mu=2 \kappa \epsilon$ finite:

$$
\begin{aligned}
\Psi(r, \phi)= & \frac{\mu}{a}+\frac{\mu}{a} \sum_{n=1}^{\infty}\left(\frac{r^{n}}{a^{n}} \cos n \phi\right)+\frac{\mu}{a} \sum_{n=1}^{\infty}\left(r^{n} a^{n} \cos n \phi\right) \\
& +\frac{\mu}{a} \sum_{n=1}^{\infty}\left[\cos n \phi\left(a^{n}+\frac{1}{a^{n}}\right)\left(r^{n}-\frac{1}{r^{n}}\right)\left(\frac{R_{o}^{2 n}}{1-R_{o}^{2 n}}\right)\right] .
\end{aligned}
$$

Replacing the first two series with their exact sums, the streamfunction can be written as 


$$
\begin{aligned}
\Psi(r, \phi)=\mu \lambda\left\{\frac{1-r \lambda \cos \phi}{1-2 r \lambda \cos \phi+\lambda^{2} r^{2}}+\frac{\lambda r \cos \phi-r^{2}}{\lambda^{2}-2 \lambda r \cos \phi+r^{2}}\right. \\
\left.\quad+\sum_{n=1}^{\infty}\left[\cos n \phi\left(r^{n}-\frac{1}{r^{n}}\right)\left(\frac{R_{o}^{2 n}}{1-R_{o}^{2 n}}\right)\left(\frac{1+\lambda^{2 n}}{\lambda^{n}}\right)\right]\right\} .
\end{aligned}
$$

From (2.8)

$$
\Psi(1, \phi)=0 \quad \text { and } \quad \Psi\left(R_{o}, \phi\right)=\mu \lambda
$$

and

$$
\Delta \Psi=-\mu \lambda .
$$

In the case of two cylinders aligned with the free stream $(\alpha=0)$, an analysis similar to the one above may be carried out with the vortex doublet rotated by $90^{\circ}$. The streamfunction for this case, a vortex doublet at $\left(\lambda^{-1}, 0\right)$ directed parallel to the imaginary axis of the transformed plane, is

$$
\begin{aligned}
& \Psi(r, \phi)=\mu \lambda\left\{\frac{-r \lambda \sin \phi}{1-2 r \lambda \cos \phi+r^{2} \lambda^{2}}+\frac{r \lambda \sin \phi}{\lambda^{2}-2 r \lambda \cos \phi+r^{2}}\right. \\
&\left.\quad+\sum_{n=1}^{\infty}\left[\sin (n \phi)\left(\frac{1}{\lambda^{n}}-\lambda^{n}\right)\left(r^{n}-\frac{1}{r^{n}}\right)\left(\frac{R_{o}^{2 n}}{1-R_{o}^{2 n}}\right)\right]\right\} .
\end{aligned}
$$

From (2.10), or by symmetry, the streamfunction difference between the cylinders for this case is

$$
\Delta \Psi=\Psi(1,0)-\Psi\left(R_{o}, \phi\right)=0 .
$$

For the general case of a uniform flow at an angle $\alpha$ to the axis joining the centres of the cylinders, $\Psi_{\alpha}(r, \phi)$ is a sum of the previous two results:

$$
\begin{aligned}
\Psi_{\alpha}(r, \phi)= & \Psi_{0}(r, \phi) \cos \alpha+\Psi_{90}(r, \phi) \sin \alpha \\
= & \mu \lambda \cos \alpha\left\{\frac{-r \lambda \sin \phi}{1-2 r \lambda \cos \phi+r^{2} \lambda^{2}}+\frac{r \lambda \sin \phi}{\lambda^{2}-2 r \lambda \cos \phi+r^{2}}\right. \\
& \left.+\sum_{n=1}^{\infty}\left[\sin (n \phi)\left(\frac{1}{\lambda^{n}}-\lambda^{n}\right)\left(r^{n}-\frac{1}{r^{n}}\right)\left(\frac{R_{o}^{2 n}}{1-R_{o}^{2 n}}\right)\right]\right\} \\
& +\mu \lambda \sin \alpha\left\{\frac{1-r \lambda \cos \phi}{1-2 r \lambda \cos \phi+\lambda^{2} r^{2}}+\frac{\lambda r \cos \phi-r^{2}}{\lambda^{2}-2 \lambda r \cos \phi+r^{2}}\right. \\
& \left.+\sum_{n=1}^{\infty}\left[\cos n \phi\left(r^{n}-\frac{1}{r^{n}}\right)\left(\frac{R_{o}^{2 n}}{1-R_{o}^{2 n}}\right)\left(\frac{1+\lambda^{2 n}}{\lambda^{n}}\right)\right]\right\} .
\end{aligned}
$$

Streamline contours for the case of a uniform stream at incidence $\alpha=-30^{\circ}$, around two cylinders of radius ratio $R_{A} / R_{B}=0.8$ and with distance between their centres equal to $2.2 R_{B}$, are compared in figure 3 with the results calculated by Lee (2000) for the same flow case.

\subsection{Cases of cylinders in relative motion}

A similar analysis can be developed when two cylinders are in relative motion to one another. Again the case of zero circulation on each of the two cylinders is considered. Attention is focused on cases in which the cylinders move with constant velocity along their line of centres, resulting in impact, and there is neither a free stream nor any 


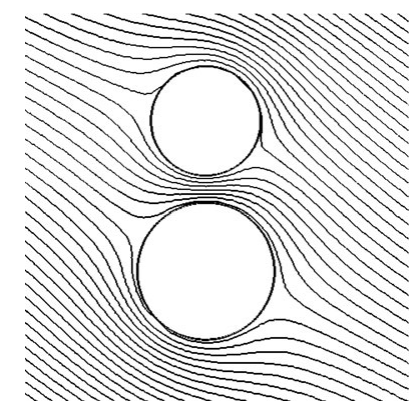

(a)

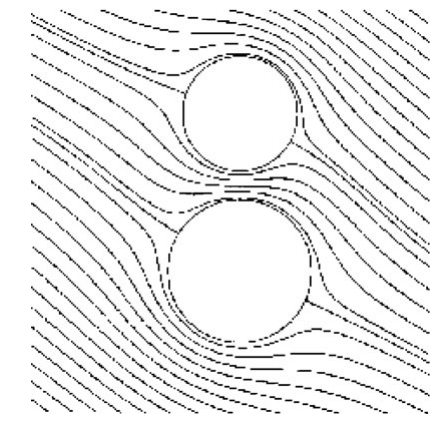

(b)

FIGURE 3. Streamlines due to a free stream in the presence of two circular cylinders, $(a)$ given by (2.11) and $(b)$ calculated by Lee (2000) (published with permission).

vortices present in the flow field. At the impulsive start of the motion, the circulation around each of the cylinders is identically zero. Assume that in a stationary fluid the $A$-cylinder (see figure 1) is stationary, and its centre is at the origin of the reference frame $(x, y)$. The second cylinder $(B$-cylinder $)$ has its centre at the point $\left(x_{B}, 0\right)$ with respect to the same reference frame. For time $t>0$, the $B$-cylinder moves with a velocity component $-U_{x, B}$ along the $x$-axis, where $U_{x, B}$ is a positive quantity. In isolation the moving circular cylinder may be represented by a source-sink dipole, whose direction is opposite to the movement of the cylinder. Again the streamfunction for this case can be obtained using a dipole method similar to that in $\S 2.4$. When this method is applied in the physical plane, the $x$-coordinate of the dipole images, is given by a continued fraction expression (see, for example, Hicks 1879). If the method of images is applied in the transformed plane, as above, the difficulty introduced by the continued fraction expressions is bypassed, and it is possible to obtain simpler expressions for the streamfunction. A dipole of strength $\mu$, at a point $\left(x_{B}, 0\right)$ in the physical plane, is transformed to a dipole of strength $\mu\left(\lambda^{2}-1\right) /\left(1-\lambda x_{B}\right)^{2}$, at the point $\left(x_{B}-\lambda\right) /\left(\lambda x_{B}-1\right)$, in the transformed plane. The dipole is inside the inner cylinder in the transformed plane and therefore, the analysis carried out in $\S 2.4$ must be modified here, since in that section the dipole was between the cylinders. The same approach is followed as before and the dipole is equated to a vortex doublet at right angles. Consider a point vortex of strength $\kappa$ at point $\zeta_{o}=R \mathrm{e}^{\mathrm{i} \phi_{o}}$ of the transformed plane. To satisfy the boundary conditions on both cylinders, an infinite series of point vortices inside the inner cylinder and outside the outer cylinder has to be constructed. The $i$ th point vortex image in the inner cylinder, has strength $\kappa$ and is located at $\zeta_{i}=R_{o}^{2 i-2} R$. Note that $i=1,2, \ldots, \infty$. The $i$ th point vortex image outside the outer cylinder has strength $-\kappa$ and lies at the point $\zeta_{i}=R_{o}^{2-2 i} R^{-1}$. Using (2.3), the streamfunction for a point vortex of strength $\kappa$ at the point $\zeta_{o}$ of the transformed plane is constructed:

$$
\begin{aligned}
\Psi(r, \phi)=-\kappa \sum_{i=1}^{\infty} \ln r & -\kappa \sum_{i=1}^{\infty} \ln R-\kappa \ln R_{o} \sum_{i=1}^{\infty}(2 \mathrm{i}-2) \\
& -\kappa \sum_{i=1}^{\infty} \sum_{n=1}^{\infty}\left[\left(R R_{o}^{2 \mathrm{i}-2}\right)^{n} \frac{\cos n\left(\phi-\phi_{o}\right)}{n}\left(r^{n}-\frac{1}{r^{n}}\right)\right] .
\end{aligned}
$$

The third-term infinite series in (2.12) diverges, but in the present case the application will be as a vortex doublet. Thus, this term is cancelled by the corresponding term for the opposite sign vortices. Using (2.12), the streamfunction of a vortex pair at 
( $R_{o} \pm \mathrm{i} \epsilon$ ), taking the limit $\epsilon \rightarrow 0$, setting $\mu=2 \kappa \epsilon$ for the dipole strength, expanding the cosine terms and cancelling equal and opposite terms, is

$$
\Psi(r, \phi)=-\frac{\mu}{R} \sum_{i=1}^{\infty} \sum_{n=1}^{\infty}\left[\left(R R_{o}^{2 \mathrm{i}-2}\right)^{n} \sin (n \phi)\left(r^{n}-\frac{1}{r^{n}}\right)\right] .
$$

The dipole strength $\mu$ is

$$
\mu=\frac{-U_{x, B} R_{B}^{2}\left(\lambda^{2}-1\right)}{\left(1-\lambda x_{B}\right)^{2}} .
$$

The streamfunction is constant on the surface of the stationary cylinder but a function of the angular coordinate $\phi$ on the moving cylinder. In the transformed plane, from (2.13),

$$
\Psi(1, \phi)=0 \quad \text { and } \quad \Psi\left(R_{o}, \phi\right)=-\frac{\mu}{R} \sum_{i=1}^{\infty} \sum_{n=1}^{\infty}\left[\left(R R_{o}^{2 \mathrm{i}-2}\right)^{n} \sin (n \phi)\left(R_{o}^{n}-\frac{1}{R_{o}^{n}}\right)\right]
$$

Summing the series, for a general point $\zeta=R_{o} \mathrm{e}^{\mathrm{i} \phi}$ on the moving cylinder,

$$
\Psi\left(R_{o}, \phi\right)=-\frac{\mu}{R}\left[\frac{R R_{o} \sin \phi}{R_{o}^{2}-2 R_{o} R \cos \phi+R^{2}}+\frac{R \sin \phi}{1-2 R \cos \phi+R^{2}}\right] .
$$

The streamfunction difference $\Delta \Psi$ between the two closest points on the two cylinders is zero by symmetry of the flow.

In addition to the above analysis for the streamfunction, the velocity potential $\Phi$ is required for the evaluation of the time-dependent force. It is convenient in this case to use a source doublet instead of a vortex doublet because of the multi-valued behaviour of the potential around a vortex. The velocity potential, due to a source dipole and its images, satisfying the boundary conditions on the cylinders is

$$
\Phi(r, \phi)=-\frac{\mu}{R} \sum_{i=1}^{\infty} \sum_{n=1}^{\infty}\left[\left(R R_{o}^{2 \mathrm{i}-2}\right)^{n} \cos n \phi\left(r^{n}+\frac{1}{r^{n}}\right)\right]
$$

The strength $\mu$ of the dipole in $(2.14)$ is $\left(\lambda^{2}-1\right) /\left(\left(1-x_{B}(t) \lambda\right)^{2}\right)$, where $x_{B}(t)$ represents the $x$-coordinate of the moving cylinder in the $z$-plane at time $t$. The velocity potentials on the surfaces of the stationary and the moving cylinders, respectively, are

$$
\begin{aligned}
\Phi(1, \phi) & =-\frac{2 \mu}{R} \sum_{i=1}^{\infty} \sum_{n=1}^{\infty}\left[\left(R R_{o}^{2 \mathrm{i}-2}\right)^{n} \cos n \phi\right], \\
\Phi\left(R_{o}, \phi\right) & =-\frac{\mu}{R} \sum_{i=1}^{\infty} \sum_{n=1}^{\infty}\left[\left(R R_{o}^{2 \mathrm{i}-2}\right)^{n} \cos n \phi\left(R_{o}^{n}+\frac{1}{R_{o}^{n}}\right)\right] .
\end{aligned}
$$

The above results have been derived for the case in which one cylinder is stationary and the other cylinder is moving relative to it in otherwise stationary fluid. Streamlines calculated from (2.13) are shown in figure 4. A fundamental case of interest, because of its symmetry, is the case in which both cylinders approach one another along their line of centres at the same speed in a stationary fluid. For potential flow this case is identical to a single cylinder impacting a plane surface. Using (2.13) and (2.14), the expressions for the streamfunction and potential on the surface of the cylinder 


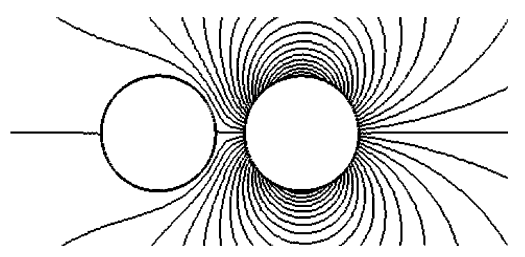

FIGURE 4. Streamlines obtained by the present method for the case of a moving cylinder approaching a fixed one.

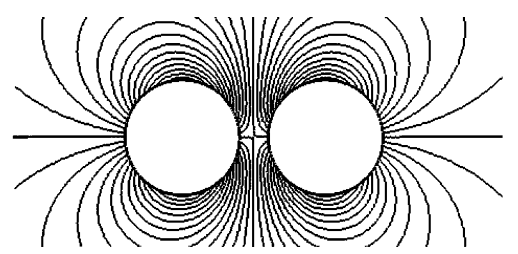

FIGURE 5. Streamlines obtained by the present method for the case of two moving cylinders approaching each other in-line with equal and opposite velocity components (frame of reference axes of symmetry).

moving in the positive $x$-direction are:

$$
\left.\begin{array}{c}
\Psi(r, \phi)=-2 \frac{\mu}{R} \sum_{i=1}^{\infty} \sum_{n=1}^{\infty}\left[\left(R R_{o}^{2 \mathrm{i}-2}\right)^{n} \sin (n \phi)\left(r^{n}-\frac{1}{r^{n}}\right)\right] \\
+U_{\infty} \frac{r\left(\lambda^{2}+1\right) \sin \phi}{\lambda^{2} r^{2}-2 \lambda r \cos \phi+1}, \\
\Phi(r, \phi)=-2 \frac{\mu}{R} \sum_{i=1}^{\infty} \sum_{n=1}^{\infty}\left[\left(R R_{o}^{2 \mathrm{i}-2}\right)^{n} \cos n \phi\left(r^{n}+\frac{1}{r^{n}}\right)\right] \\
++_{\infty} \frac{\lambda r^{2}-r\left(\lambda^{2}+1\right) \cos \phi+\lambda}{\lambda^{2} r^{2}-2 \lambda r \cos \phi+1} .
\end{array}\right\}
$$

Streamlines calculated from (2.15) are shown in figure 5. Results can similarly be derived for the case in which the relative motion between two cylinders is perpendicular to their line of centres. If the moving cylinder, centred at the point $\left(x_{B}, 0\right)$, moves with a velocity component $-U_{y}$ in the $y$-direction only $\left(U_{y}>0\right)$, while the other is held stationary, an analysis similar to the one above shows that

$$
\Psi(r, \phi)=-\frac{\mu}{R} \sum_{i=1}^{\infty} \sum_{n=1}^{\infty}\left[\left(R R_{o}^{2 \mathrm{i}-2}\right)^{n} \cos n \phi\left(r^{n}-\frac{1}{r^{n}}\right)\right],
$$

where $\mu=-U_{y} R_{B}^{2}\left(\lambda^{2}-1\right) /\left(1-\lambda x_{B}\right)^{2}$. Carrying out the double summation $\Psi(1)=0$ and

$$
\Psi\left(R_{o}, \phi\right)=-\frac{\mu}{R}\left[\frac{R\left(R_{o} \cos \phi-R\right)}{R_{o}^{2}-2 R_{o} R \cos \phi+R^{2}}+\frac{R(\cos \phi-R)}{1-2 R \cos \phi+R^{2}}\right],
$$

where $\phi$ is the angular coordinate on the moving cylinder in the transformed plane. The general case in which one cylinder moves at constant velocity in any direction and the other cylinder is fixed can be derived as a linear sum of the two special cases above. 


\section{Forces induced on each of two circular cylinders approaching in-line}

First we consider the case in which one cylinder remains fixed and the other moves towards it. The pressure at an arbitrary point, in an inviscid flow field, with respect to an inertial reference frame is given by the Bernoulli equation

$$
\frac{p}{\rho}=-\frac{\partial \Phi}{\partial t}-\frac{1}{2} q^{2}+C(t)
$$

where $q=\sqrt{u^{2}+v^{2}}$, and $C(t)$ is a spatially constant term. If $q$ denotes the absolute velocity of the fluid at a point on a moving cylinder (centre at $x_{o}=0$ at time of evaluation), which is moving with velocity $\left(U_{x}, U_{y}\right)$, the Bernoulli equation becomes

$$
\frac{p}{\rho}=-\frac{\partial \Phi}{\partial t}-\left.\frac{\partial \Phi}{\partial x_{o}} \frac{\mathrm{d} x_{o}}{\mathrm{~d} t}\right|_{x_{o}=0}-\left.\frac{\partial \Phi}{\partial y_{o}} \frac{\mathrm{d} y_{o}}{\mathrm{~d} t}\right|_{y_{o}=0}-\frac{1}{2} q^{2}+C(t) .
$$

If the cylinder moves only with an $x$-velocity component $U_{x}$, then (3.1) simplifies to

$$
\frac{p}{\rho}=-\frac{\partial \Phi}{\partial t}-U_{x} u-\frac{1}{2} q^{2}+C(t),
$$

where $u=\partial \Phi / \partial x$. The part $F_{x}^{I}$ of the force component $F_{x}$ in the positive $x$-direction, resulting from the time derivative of the velocity potential, can be evaluated by using the extension of Blasius's theorem for a moving cylinder:

$$
F_{x}^{I}=-\mathrm{i} \rho \frac{\partial}{\partial t} \oint_{C} w \mathrm{~d} z
$$

where $C$ is the surface of the cylinder. Transforming the integration parameter from $z$ to $\zeta$, the integration contour $C_{A}$ of the stationary cylinder in the physical plane transforms to the contour $C_{A}^{\prime}(r=1)$ in the $\zeta$-plane; the part contributed by the $\partial \Phi / \partial t$ term on the stationary cylinder is given as follows:

$$
F_{x}^{I}=-\mathrm{i} \rho \oint_{C_{A}} \frac{\partial w(z)}{\partial t} \mathrm{~d} z=\mathrm{i} \rho \frac{\partial}{\partial t}\left(\frac{\lambda^{2}-1}{\lambda^{2}} \oint_{C_{A}^{\prime}} \frac{w(\zeta)}{\left(\zeta-\lambda^{-1}\right)^{2}} \mathrm{~d} \zeta\right) .
$$

The integrand $f(\zeta)=w(\zeta) /\left(\zeta-\lambda^{-1}\right)^{2}$ of (3.2) is evaluated using the residue theorem. Expressing $w$ in terms of its singularities, $f(\zeta)$ is written as

$$
f(\zeta)=\frac{w(\zeta)}{\left(\zeta-\lambda^{-1}\right)^{2}}=\left[\sum_{i=1}^{\infty}\left(\frac{\mu R_{o}^{2 \mathrm{i}-2}}{\zeta-R R_{o}^{2 \mathrm{i}-2}}\right)-\sum_{i=1}^{\infty}\left(\frac{\mu R^{-2} R_{o}^{2-2 \mathrm{i}}}{\zeta-\left(R R_{o}^{2 \mathrm{i}-2}\right)^{-1}}\right)\right] \frac{1}{\left(\zeta-\lambda^{-1}\right)^{2}} .
$$

The singularities are at points $\zeta_{1}=R R_{o}^{2 \mathrm{i}-2}, \zeta_{2}=\left(R R_{o}^{2 \mathrm{i}-2}\right)^{-1}$ and $\zeta_{3}=\lambda^{-1}$. Only the first and third set of points are inside the contour $C_{A}^{\prime}$ of the stationary cylinder in the $\zeta$ plane. Therefore, only the residues at these singularity point sets have to be evaluated to obtain the force. Combining the expressions for the residues and substituting them in (3.2), the $F_{x}^{I}$ part of the force on the stationary cylinder is obtained as

$$
F_{x}^{I}=-2 \pi \rho \frac{\partial}{\partial t}\left\{\frac{\lambda^{2}-1}{\lambda^{2}}\left[\sum_{i=1}^{\infty} \operatorname{Res}\left[f(\zeta), \zeta_{3}\right]+\sum_{i=1}^{\infty} \operatorname{Res}\left[f(\zeta), \zeta_{1}\right]\right]\right\} .
$$

The same part of the force on the moving cylinder is given by

$$
F_{x}^{I}=-\mathrm{i} \rho \oint_{C_{B}} \frac{\partial w(z)}{\partial t} \mathrm{~d} z=-\mathrm{i} \rho \oint_{C_{B}^{\prime}} \frac{\partial w(\zeta)}{\partial t} \frac{\mathrm{d} z}{\mathrm{~d} \zeta} \mathrm{d} \zeta .
$$




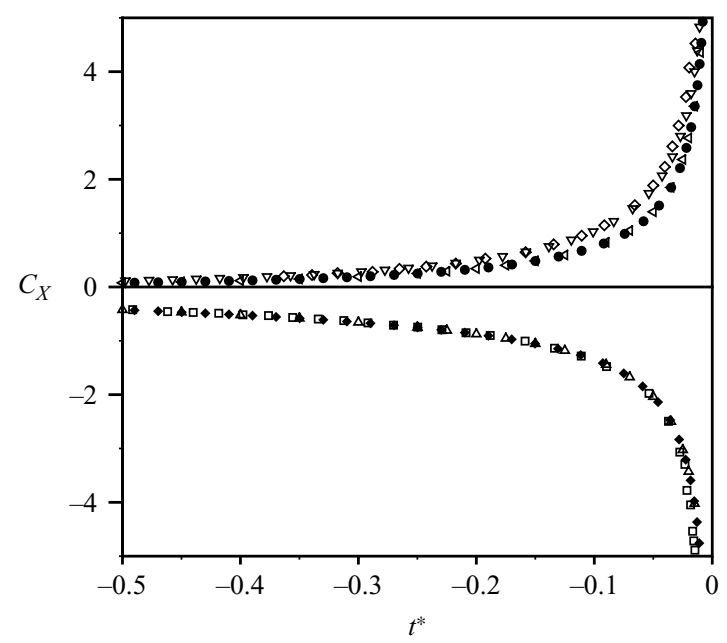

FIGURE 6. Inviscid force coefficient $C_{X}$ when a moving cylinder impacts a stationary cylinder. -, Present method moving; $\nabla,\left(U_{x} \partial \Phi / \partial x\right.$ not included $)$; $\diamond$, Present method stationary; $\diamond$, Wang (2004) moving; $\square$, Wang (2004) stationary; $\triangleleft$, Landweber et al. (1991) moving; $\triangle$, Landweber et al. (1991) stationary.

The transformed contour of the moving cylinder in the $\zeta$-plane $C_{B}^{\prime}$ is an expanding circle, i.e. a moving boundary. Therefore, the order of the time derivative and the spatial integration cannot be simply interchanged. As the boundary expands with time, the singularity points $\left(R R_{o}^{2 \mathrm{i}-2}, 0\right)$ also move, but they remain enclosed by the expanding contour $C_{B}^{\prime}$. Appendix shows that the two operations may be interchanged. The evaluation of this part of the force on the moving cylinder is now greatly simplified. Using a result obtained previously when evaluating the sum of the residues at points $\left(R R_{o}^{2 i-2}, 0\right), F_{x}^{I}$ for the moving cylinder is given by

$$
F_{x}^{I}=2 \pi \rho \frac{\partial}{\partial t}\left[\frac{\lambda^{2}-1}{\lambda^{2}} \sum_{i=1}^{\infty} \operatorname{Res}\left[f(\zeta), \zeta_{1}\right]\right] .
$$

$F_{x}^{I}$ was also calculated as a check by integrating $\partial \Phi / \partial t$ numerically around the cylinder, and the result was shown to agree with the above result.

The contribution to the pressure term due to the velocity in Bernoulli's equation

$$
-\frac{1}{2} \rho q^{2}=-\frac{1}{2} \rho\left|\frac{\mathrm{d} w}{\mathrm{~d} z}\right|^{2}
$$

is now calculated in terms of the speed $|\mathrm{d} w / \mathrm{d} z|$ on the cylinders' surfaces, using finite difference differentiation and numerical integration around the $C_{i}$ boundary:

$$
F_{x}^{I I}=-\frac{1}{2} \rho \oint_{C_{i}} q^{2} \cos \theta \mathrm{d} \theta .
$$

The cross-term in the Bernoulli equation for a moving body $u U_{x}$ is also integrated numerically around the cylinder. Combining the three terms of the force gives the total force on each cylinder. The force coefficient on each cylinder in the $x$-direction $C_{x}=F_{x} /\left(0.5 \rho U_{2}^{2} D\right)$ is plotted against the dimensionless time $t^{*}=U_{x, B}\left(t-t_{\text {impact }}\right) / D$, in figure 6, where $t_{\text {impact }}$ denotes the time at which impact occurs and $D$ the cylinder's diameter. In this figure the results by Wang (2004) and Landweber et al. (1991) for the total force on the cylinders are also presented for comparison. 


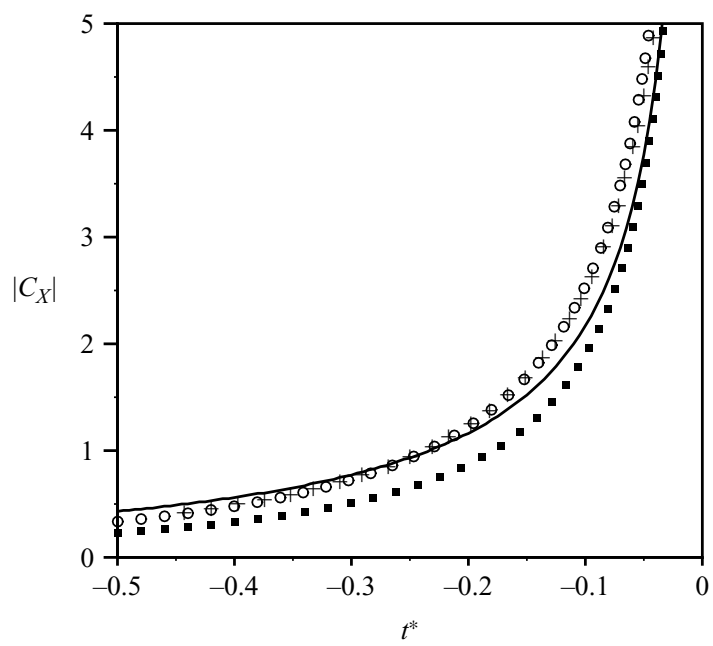

FigURE 7. Inviscid force coefficient $C_{X}$ when two moving cylinders impact symmetrically in

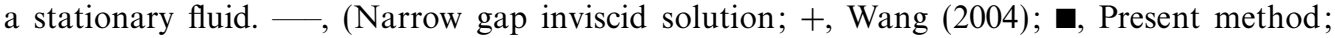
$\bigcirc,\left(U_{x} \partial \Phi / \partial x\right)$ not included $)$.

The three sets of results all agree exactly for the stationary cylinder. On the other hand, for the force on the moving cylinder there is a disagreement. While the present results agree with?, the curve predicted by Wang (2004) is not the same. The reason appears to be omission of the cross-term contribution $U_{x} \partial \Phi / \partial x$ to $\partial \Phi / \partial t$ from the spatial variation of the velocity potential on a moving cylinder as is shown by agreement between Wang's curve and the present computations when this term is omitted.

In the case of both cylinders moving towards each other symmetrically, the same procedure can be followed to evaluate the inviscid force from the Bernoulli equation using the results for $\Phi$ and $\Psi$ obtained in $\S 2.5$. Figure 7 shows the results for the force coefficient predicted by the present method and a second set of points evaluated without including the above cross-term, $U_{x} \partial \Phi / \partial x$, which agrees with the results by Wang (2004). Figure 8 shows the forces plotted on logarithmic scales for both cases. These plots suggest that for all these cases the inviscid force is singular at impact, tending to infinity like $t^{*-1 / 2}$, as $t^{*} \rightarrow 0$.

\subsection{A narrow gap analysis}

An approximate narrow gap inviscid analysis may be carried out, just before impact, to establish the behaviour of the forces on the cylinders with the time to impact $t^{*} \rightarrow 0$. Consider first the case of $(a)$ two equal-diameter cylinders moving towards each other along their line of centres, with equal and opposite velocities $U_{x}$ in an otherwise stationary fluid $\left(U_{\infty}=0\right)$. In the diagram shown in figure 9, which shows half the gap, of total width $b_{o}(t)$ between the cylinders, $\pm U_{x}$ is the speed of each cylinder. In this approximate analysis $v$, the speed of the squeezed flow in the $y$-direction, is assumed to be uniform across the gap, as is the pressure $p$; i.e. a quasi-one-dimensional analysis is assumed. By continuity $v=2 U_{x} y / x_{o}$, where $x, y_{o}$ denote the coordinates of the cylinder's surface. From this, the time derivative of the velocity potential in the gap can be obtained as a function of $\theta$, the angular coordinate around either cylinder, 


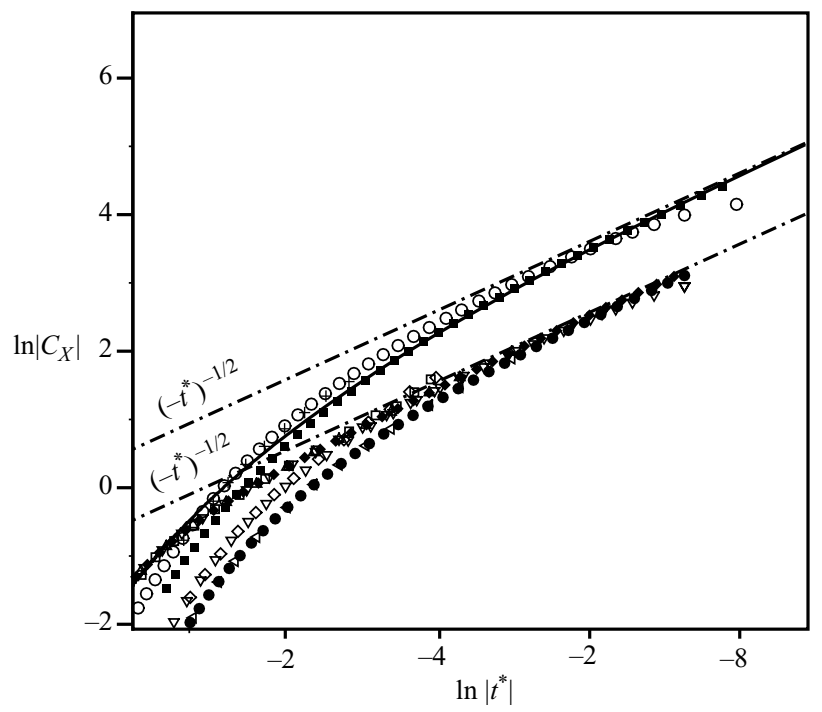

FIGURE 8. Logarithmic plot of $C_{X}$. For cases shown in figures 6 and 7. Symbols as in those figures.

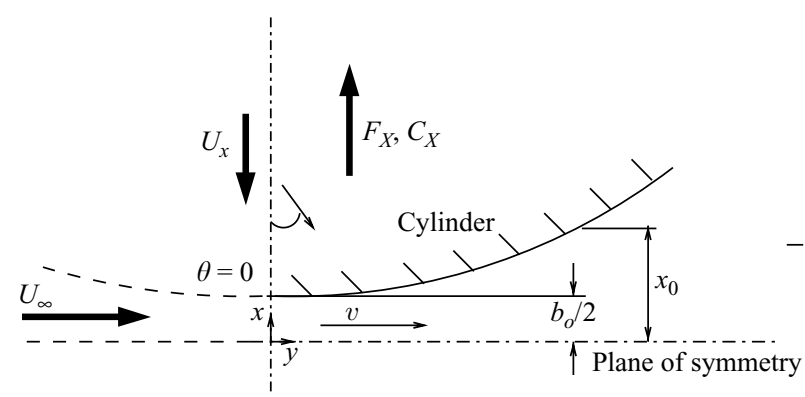

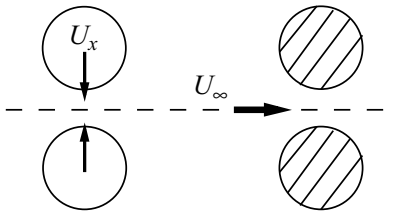

(a)

(b)

FiguRE 9. Schematic diagram for the narrow gap solution using the Euler equations.

as

$\frac{\partial \Phi}{\partial t}=U_{x}^{2} \int_{0}^{\theta} \frac{\sin \phi \cos \phi}{(\sigma-\cos \phi)^{2}} d \phi=\frac{-U_{x}^{2} \cos \theta}{\sigma-\cos \theta}-U_{x}^{2} \ln (\sigma-\cos \theta)+\frac{U_{x}^{2}}{\sigma-1}+U_{x}^{2} \ln (\sigma-1)$,

where $\sigma=1-2 t^{*}$ is a parameter based on the dimensionless time $t^{*}$. The pressure is calculated from Bernoulli's equation

$$
\frac{p}{\rho}=\frac{p_{o}}{\rho}-\frac{\partial \Phi}{\partial t}-\frac{1}{2} v^{2}
$$

where $p_{o}$ is a constant. The singular part of the force, as the gap closes, arises from the pressure in the narrowest part of the gap. Therefore the singular part of the force will not be affected by the location at which it is assumed that pressure is equal to ambient $p_{a}$. For simplicity this is assumed to be at the ends of the gap: $\theta= \pm \pi / 2$. It is further assumed that $p=p_{a}$ for $\theta>\pi / 2$ or $<-\pi / 2$, i.e around the whole base region of each cylinder. The surface pressure is therefore given by 


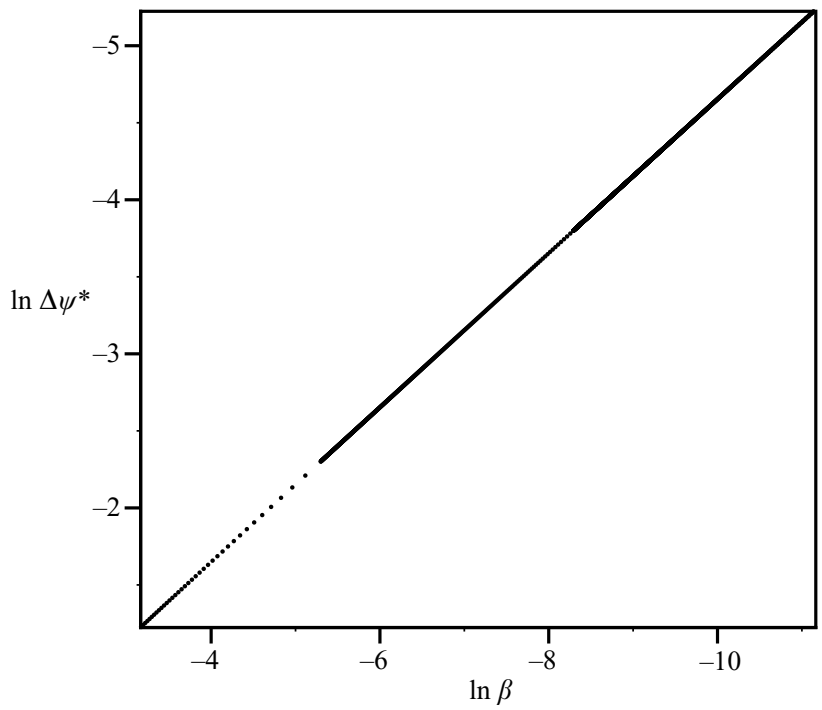

FiguRE 10. Logarithmic plot of the dimensionless streamfunction flux between two cylinders in a free stream transverse to the line of centres as a function of the dimensionless distance between them.

$$
\left.\begin{array}{cc}
\frac{p-p_{a}}{\rho}= & -\frac{U_{x}^{2}}{\sigma-1}-U_{x}^{2} \ln (\sigma-1)+\frac{U_{x}^{2} \cos \theta}{\sigma-\cos \theta}+U_{x}^{2} \ln (\sigma-\cos \theta), \\
& -\frac{U_{x}^{2} \sin ^{2} \theta}{2(\sigma-\cos \theta)^{2}} \quad \text { when }|\theta| \leqslant \frac{\pi}{2} \\
0 & \text { when }|\theta|>\frac{\pi}{2}
\end{array}\right\}
$$

Now (3.3), which gives the pressure on the cylinder, is integrated to give an estimate of the force on the cylinder. Thus

$$
C_{X}=\frac{1}{\sigma^{2}}+\int_{0}^{\pi / 2}\left[\frac{\sigma\left(\sigma^{2}-1\right)}{(\sigma-\cos \phi)^{2}}+\frac{\sigma^{2}-1}{\sigma-\cos \phi}-3 \cos \phi-2 \sigma\right] \mathrm{d} \phi .
$$

Carrying out the integration gives the in-line force coefficient:

$$
C_{X}=\frac{1}{\sigma^{2}}-3-\pi \sigma+\frac{2\left(2 \sigma^{2}-1\right)}{\sqrt{\sigma^{2}-1}} \tan ^{-1} \sqrt{\frac{\sigma+1}{\sigma-1}} .
$$

The inviscid force from this approximate analysis is plotted in figures 7 and 8 , showing good agreement with the exact computed results as $t^{*} \rightarrow 0$. Taking the largest order term in (3.4) shows that

$$
C_{X} \rightarrow(\pi / 2)\left(-t^{*}\right)^{-1 / 2} \quad \text { as } t^{*} \rightarrow 0 .
$$

A similar narrow gap analysis can be applied to $(b)$ two stationary cylinders, separated by a narrow gap $\beta=b_{o} / D$, in a free stream $U_{\infty}$ transverse to the line of centres (figure 9). In this case the volume flow between the cylinders must be determined. The difference in values of the streamfunctions on the surfaces of the two cylinders, $\Delta \Psi$, can be shown for the case in which there is zero circulation on each of the cylinders, as assumed here, to tend to zero as the gap is made as small as $\beta^{1 / 2}$. Figure 10 shows a logarithmic plot of the dimensionless $\Delta \Psi^{*}$ computed from (2.9) as a function of dimensionless gap size $\beta$, down to very small gap sizes. It can 
be seen that a close approximation is given by

$$
\Delta \Psi^{*}=\frac{\Delta \Psi}{U_{\infty} D} \simeq \sqrt{2} \beta^{1 / 2} .
$$

Using this to give the volume flow through the gap and the same narrow gap approximation as above shows that in this case

$$
C_{X}=-\frac{\Delta \Psi^{* 2}}{\left(\sigma^{2}-1\right)}\left(\frac{1}{\sigma^{2}}+\frac{2}{\sqrt{\sigma^{2}-1}} \tan ^{-1} \sqrt{\frac{\sigma+1}{\sigma-1}}\right),
$$

which indicates that $C_{X} \rightarrow \pi \beta^{-1 / 2} / \sqrt{2}$ as $\beta \rightarrow 0$. The inviscid forces on the cylinders in this case are attractive, which will be seen to be the opposite of the case for viscous flow. For the general case, the two narrow gap flow fields may be combined to give the result for a pair of cylinders moving towards each other at speed $\pm U_{x}$ in a transverse free stream $U_{\infty}$. The velocity $v$ in the gap is antisymmetric with respect to $\theta$ in case $(a)$ but symmetric with respect to $\theta$ in case $(b)$. Therefore, while the squared terms from each velocity distribution in Bernoulli's equation will contribute to the force coefficient $C_{X}$, the cross-product term from the two velocity distributions $(a) \cdot(b)$, being antisymmetric, will not. Also the potential having the opposite symmetry to the velocity with respect to $\theta$, the $\partial \Phi / \partial t$ term for the now unsteady flow field of case (b) is antisymmetric and does not contribute, whereas in case $(a)$ it does. This shows that for the largest terms, in the limit as the gap closes, the asymptotic result is

$$
\lim _{t^{*} \rightarrow 0} C_{X}=\frac{\pi}{2}\left(\frac{\gamma^{2}-1}{\gamma^{2}+1}\right)\left|t^{*}\right|^{-1 / 2}+O(1),
$$

where $\gamma$ is the velocity ratio $U_{x} / U_{\infty}$. Hence, the singularity in the force coefficient changes sign for inviscid flow at a velocity ratio $U_{x} / U_{\infty}=1$, at which value the force remains finite right up to impact $C_{X}\left(t^{*}=0\right)=-(\pi / 2+3 / 2)$. Figures 11 and 12 show values of the inviscid force coefficient $C_{X}$ for a range of values of the velocity ratio $U_{x} / U_{\infty}$, computed by combining the image system solutions of $\S 2.4$ and the steady gap flow above. The results are compared with the narrow gap analysis. In the case $U_{x} / U_{\infty}=1$, for accuracy, three computations with different time steps, taken to very small values, are shown to approach the finite force limit at impact.

\section{Viscous flow}

\subsection{Overview of the numerical method}

In this section the viscous impact of two circular cylinders is investigated, using an Eulerian-Lagrangian numerical code (Willden \& Graham 2001) to solve the twodimensional streamfunction-vorticity formulation of the Navier-Stokes equations:

$$
\begin{gathered}
\nabla^{2} \Psi=-\omega \\
\frac{\partial \omega}{\partial t}+u \frac{\partial \omega}{\partial x}+v \frac{\partial \omega}{\partial y}=v \nabla^{2} \omega .
\end{gathered}
$$

As before $\Psi$ denotes the streamfunction; $\omega$ is the vorticity of the flow field; $u, v$ are the $x$ and $y$ velocity components of the fluid; and the centres of the cylinders lie on the $x$-axis. The method used solves (4.1) and (4.1) on an unstructured mesh of triangles fitted to the bodies as shown in figure 13 over a sequence of time steps. A split of (4.2) into diffusion and convection sub-steps within each time step is carried out. 


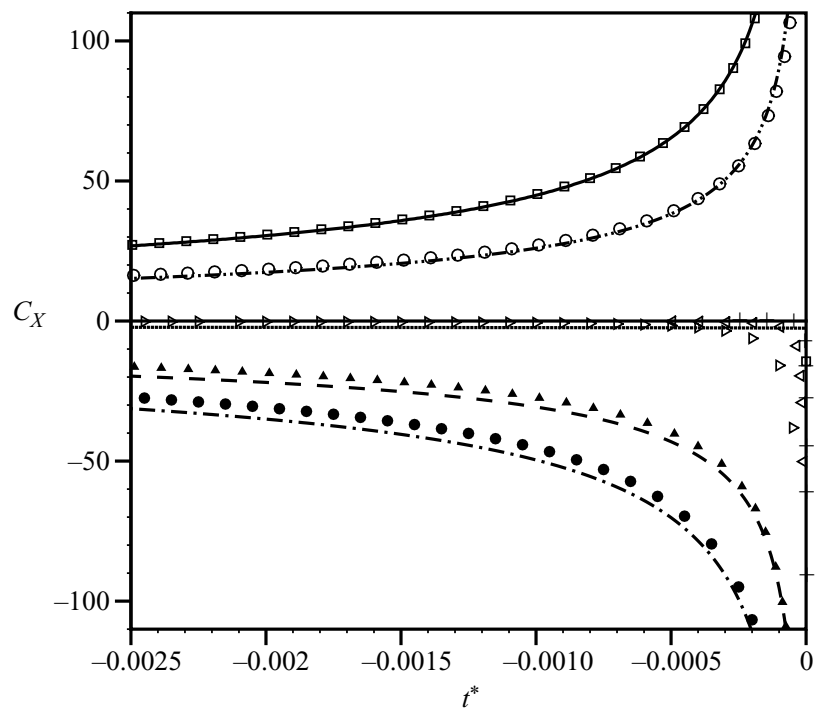

FIGURE 11. Force coefficient $C_{X}$ as cylinders approach impact in the presence of a cross flow $U_{\infty}\left(\Delta t^{*}=5 \times 10^{-5}\right.$ except as shown $)$.,$\left(U_{x} / U_{\infty}=0\right) ; \boldsymbol{\Delta},(0.5) ; \triangleright,\left[U_{x} / U_{\infty}=1.0 ; \triangleleft\right.$, $\left.\left(\Delta t^{*}=5 \times 10^{-6}\right) ;+,\left(\Delta t^{*}=5 \times 10^{-7}\right)\right] ; \bigcirc,(2.0) ; \square,(\infty)$. Narrow gap solutions: $-\cdot-\cdot-$, $\left(U_{x} / U_{\infty}=0\right) ;---,(0.5), \cdots(1.0) ;-\cdots-\cdots-,(2.0) ;-,(\infty)$.

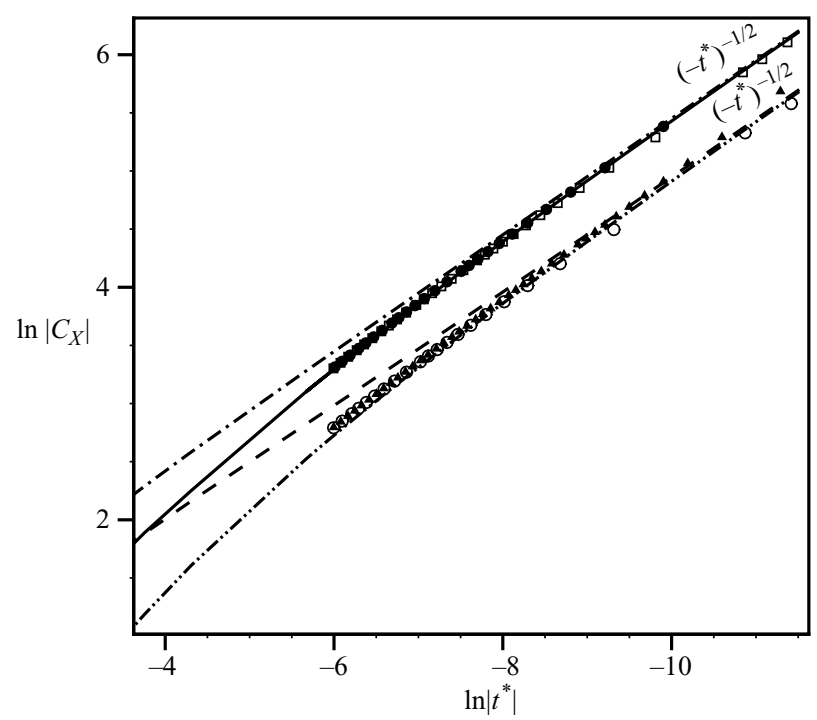

FIGURE 12. Logarithmic plot of the force coefficient $C_{X}$ for cylinders impacting in a transverse flow. Symbols as in figure 11 .

First, in each time step, the Poisson's equation is solved using the Galerkin weighted residual finite element method, applying at this step only the no-penetration boundary condition on the cylinders' surfaces. This is applied as a Dirichlet condition on the streamfunction $\Psi$, using the results for the streamfunction difference $\Delta \Psi$ between the cylinders obtained in the previous sections. Since $\Psi$ contains an arbitrary constant, the value $\Psi=0$ can be set at a reference point on a boundary. In the present case this point was taken to be on the surface of one of the cylinders, with $\Psi=0$ over 


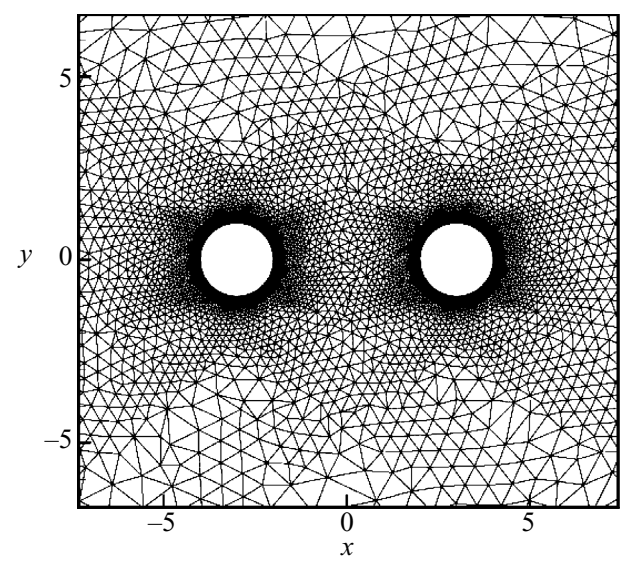

FIGURE 13. Unstructured mesh used for the case of plane impact.

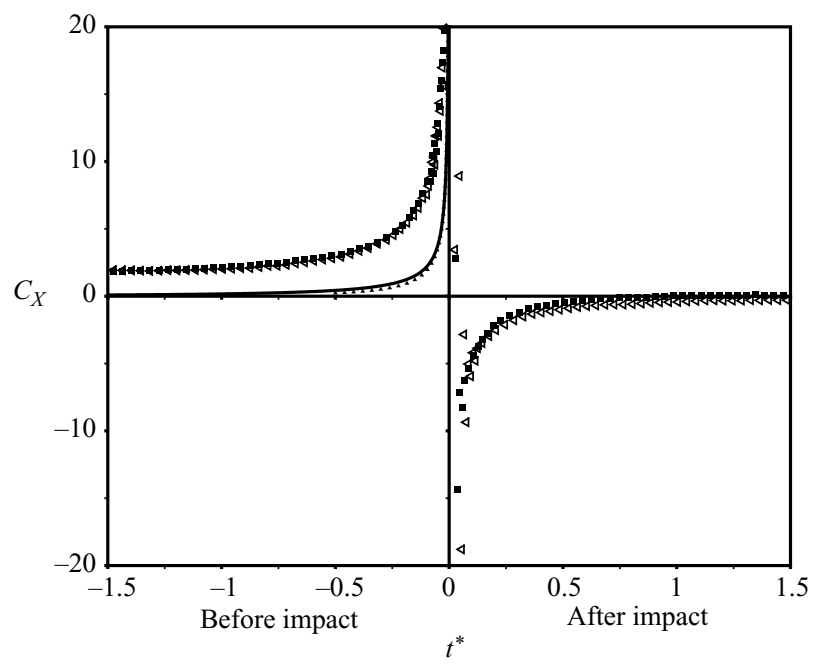

FIGURE 14. Effect of $L$ on $C_{X} . R e=100, \triangleright,(L / D=3) ; \mathbf{\square},(L / D=5) ; \boldsymbol{\Delta},(R e=\infty$, inviscid computations); - Narrow gap inviscid solution.

the whole surface if it was stationary in the frame of reference used. From the earlier mesh-free analysis of $\S 2,(2.7),(2.9)$ and (2.15) then provide the value of $\Psi$ on the other cylinder respectively for the effects of vortices in the flow field, the free stream and the relative motion of the cylinders. The value of $\Psi$ also calculated in $\S 2$ for the effect of cylinder circulation is not required, since each cylinder's circulation in no-slip viscous flow is carried in the boundary layers, which together with the shed vorticity is represented by the discrete vortices in this method. Similarly, the value of $\Psi$ on the outer boundary of the mesh is calculated from (2.5), (2.11) and (2.15). This procedure was followed to calculate all the boundary conditions for $\Psi$ at this stage in each time step except that it was found that including the contribution (2.5) for the effect of the vortices in the flow field on the outer boundary condition led to a computational instability. It was therefore omitted from the outer boundary condition, since the total circulation of the flow in the computation domain remains close to zero throughout 
the flow development, and the outer boundary condition was calculated from (2.11) and (2.15) only.

Then the change in vorticity due to diffusion is computed by applying a Galerkin weighted residual finite element method to solve the diffusion sub-step of (4.2)

$$
\frac{\partial \omega}{\partial t}=v \nabla^{2} \omega
$$

using either backward Euler or second-order Crank-Nicholson time integration. The boundary conditions applied on $\omega$ are of the Neumann type (Koumoutsakos, Leonard \& Pépin (1994) and enforce the no-slip condition on the surfaces of the bodies. The kinematic relations between velocity and vorticity (derivatives of (4.1)),

$$
\begin{gathered}
\nabla^{2} u=-\frac{\partial \omega}{\partial y}, \\
\nabla^{2} v=\frac{\partial \omega}{\partial x}
\end{gathered}
$$

are then solved on the mesh to evaluate the velocity field at the intermediate sub-step. At this stage the vorticity field adjacent to the body surfaces has been calculated for the current step by the solution of (4.3), shedding new vorticity as necessary. Hence both no-slip and no-penetration boundary conditions $(u=0$ and $v=0$ for stationary cylinders) can be applied for the solutions of (4.4) and (4.5). In this procedure an initial solution of (4.1) for the streamfunction provides a lower accuracy velocity field for the vorticity boundary condition to equation (4.3), after solving which, equations (4.4) and (4.5), with the no-slip and no-penetration boundary conditions, can be solved to provide a more accurate, corrected solution of the velocity field. Results have been compared with and found to be more accurate than solutions of either the streamfunction field (i.e. omitting the step of solving (4.4) and (4.5)) or the velocity field omitting pre-calculation of the streamfunction (4.1). The convection stage of (4.2) is solved by Lagrangian particle tracking. The nodal circulation changes calculated on the mesh during the diffusion sub-step are assigned to point vortices, which are then convected according to the local velocity (Lagrangian sub-step), using a first-order scheme. The method of solution is then repeated for the next time step. Because of the relative motion in the present study, a moving mesh is necessary for the Eulerian part of the solution, but the Lagrangian convection procedure is only trivially affected. The mesh is continually distorted to follow the moving boundaries, using the spring-segment method. For large boundary displacements, the mesh may become highly distorted, and this clearly affects the accuracy of the solution. To overcome this difficulty, a re-meshing is performed when necessary, based on a constrained Delaunay triangulation algorithm which reassigns the connectivity of the node points, forming the mesh. The small discontinuities particularly visible in logarithmic plots of the force (such as in figure 17) are due to re-meshing. The computations were carried out with a domain size $50 \mathrm{D}$ wide and $85 \mathrm{D}$ long.

\subsection{Plane impact of two cylinders with zero free stream}

The plane impact of two circular cylinders of equal diameter immersed in incompressible viscous fluid at rest at infinity is considered. The critical parameters of impact to be studied are the ratio of the initial distance between the cylinders at the start of their motion to their diameter, the Reynolds number based on a reference velocity, usually the velocity of the moving cylinder and the ratios of the relative velocities of the cylinders to that of the fluid. The two cylinders are assumed to start 


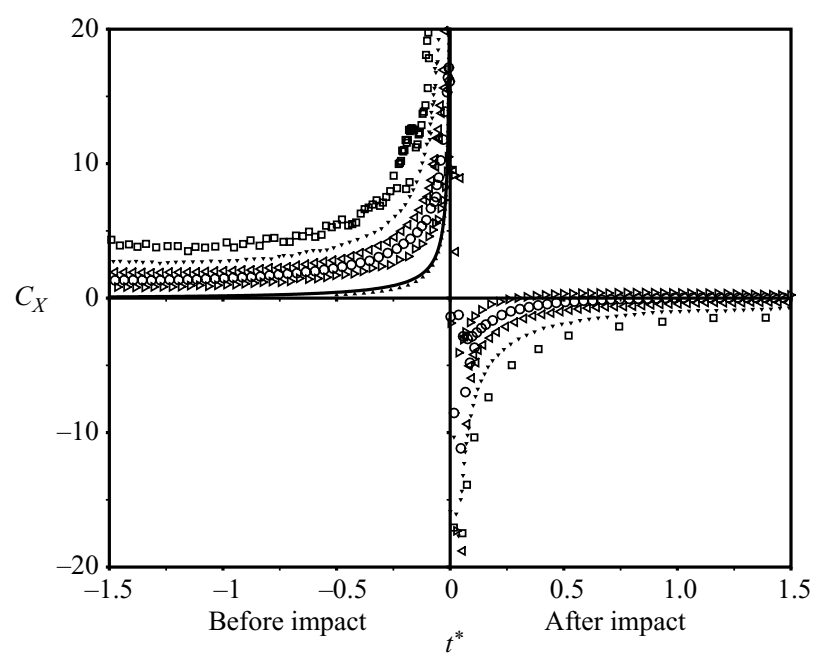

Figure 15. Effect of $R e$ on $C_{X} . L / D=3: \square,(R e=25) ; \nabla,(R e=50) ; \triangleleft,(R e=100)$; , $(R e=200) ; \triangleright,(R e=500) ; \boldsymbol{\Lambda},(R e \rightarrow \infty$, Method of images $) ;-$, Narrow gap inviscid solution.

impulsively and move towards each other along their line of centres with the same constant speed. At impact they are assumed to stop moving instantaneously and remain stationary and in contact thereafter. This is the simplest case for two cylinders resulting in an impact. It is idealized in that real impacts are invariantly threedimensional and involve structural deformations and some degree of elastic rebound, all of which greatly complicate the analysis and are not considered here. As the two cylinders approach each other finally towards impact, the gap between them becomes very small. Resolution of this flow requires locally very small mesh elements and also very small time steps. At impact the boundaries touch, and therefore $\Delta \Psi^{*}$ between the cylinders becomes zero. The inviscid computation of $\Delta \Psi^{*}$ for two cylinders impacting shows that $\Delta \Psi^{*} \simeq \sqrt{2} \beta^{1 / 2}$. The infinite rate of change as $\beta \rightarrow 0$ may be one source of the numerical oscillations observed in the viscous flow computations after impact. In figures 14 and 15 the resulting force coefficient acting along the line of centres (repulsion positive) on either cylinder is plotted versus the dimensionless time coefficient. The initial separation distance between centres $L$ is relatively small ( $3 D$ or $5 D$ ), and the Reynolds number $R e$ is also small. Hence the flows remain essentially symmetric throughout. In these plots, the inviscid force derived in the previous sections for the same motion is also plotted, as is the approximate narrow gap inviscid solution.

Figure 14 shows the result of changing the initial separation $L$ between the cylinders. $L$ is constrained by the need to limit computation times. It is clear from the results shown for $L=3 D$ and $5 D$ that as long as the initial separation is sufficient at the start for the cylinders to be effectively independent and not so large that natural flow asymmetry develops, the parameter $L / D$ does not significantly affect the flow-induced force on the cylinders. Figure 15 shows the effect of changing the Reynolds number. The repelling force coefficient before impact on each cylinder, as would be expected due to the increased resistance to the transverse flow in the gap, is larger than the inviscid force and decreases towards the inviscid force with increasing Reynolds number. 
All the computed results show reduction in the rate of increase of force as $t^{*} \rightarrow 0$. At the highest Reynolds number computed, $R e=500$, the $C_{X}$ curve ultimately falls below the inviscid force curve at the smallest $t^{*}$. The computed results in the final stages of closure of the gap become progressively less accurate due to mesh resolution, because of constraints on re-meshing. It is therefore believed that the reduction in the rate of increase of the force is a numerical artefact. If so the computed results suggest that $C_{X}$ (viscous) is singular at contact with the power dependence of $t^{*-1 / 2}$ similar to the inviscid force. The limit of infinite force is supported by the work of Brenner (1961) for the weaker case of a sphere impacting a plane, showing that the force is singular in that case, but Stimson \& Jeffery (1926) for the case of two spheres impacting indicate a finite limit. Christensen (1962) has shown that within the assumptions of lubrication theory, which ignores the inertia terms, the force between two cylinders remains finite at contact. In the inviscid case it is the inertia terms which dominate and cause the singularity at impact. The viscous shear force changes the flow profile in the gap, adding resistance and hence increasing the value of pressure gradient along the gap above that required to accelerate the bulk flow which is unchanged. If one now considers the motion of two free cylinders approaching one another at high Reynolds number and decelerating due to the force induced between them and take the above asymptotic inviscid fluid force for constant cylinder velocity, $C_{X} \sim(\pi / 2)\left(-t^{*}\right)^{-1 / 2}$, as a guide, the deceleration of each cylinder is given by

$$
\frac{\mathrm{d}^{2} x}{\mathrm{~d} t^{2}}=-\frac{\pi \rho D^{3 / 2}}{4 m}\left|\frac{\mathrm{d} x}{\mathrm{~d} t}\right|^{3 / 2}\left(t_{\text {impact }}-t\right)^{-1 / 2},
$$

where $m$ is the mass of the cylinder per unit length. Integrating this equation, ignoring the effect of cylinder deceleration on the flow, shows that the ratio of the velocity of the cylinders at impact $U_{x}^{\prime}$ to their velocity at the start of the motion $U_{x}$ is $U_{x}^{\prime} / U_{x} \geqslant\left[1+(L / D)^{1 / 2} / m^{*}\right]^{-2}$, where $m^{*}$ is the mass ratio of the cylinder to the mass of fluid it displaces, and $L$ is the initial gap between the cylinders at the point at which they have the 'starting' velocity $U_{x}$. Inserting typical values such as $m^{*}=3$ and $L / D=1$, for example for marine riser pipes, shows that the fluid force during impact is likely to slow the cylinders significantly (in this case $U_{x}^{\prime} / U_{x} \gtrsim 0.56$ ) but not to a negligible impact velocity. In figure 16 contour plots of the dimensionless vorticity $\omega^{*}=\omega D / U_{x}$, for the case in which $L=5 D, R e=100$, are shown for various times ranging from shortly before impact to sometime after. Immediately after impact, secondary boundary layers of opposite vorticity are created beneath the original boundary layers formed around the cylinders during the motion prior to impact. Also after impact, primary vortices formed from the initial wakes and boundary layers move around the bodies and convect transversely parallel to the transverse symmetry plane. Similarly secondary vortices are formed by rolling up of the separating secondary boundary layers. The self-induced velocity field of each primary vortex pair is such as to cause it to convect outwards along the plane of symmetry normal to the cylinder motion. The opposite is true of the secondary vortices which are nonetheless convected outwards by the stronger primary field. Figure 17 shows that after impact, if the cylinders remain in contact and motionless as has been assumed for these calculations, the force reverses to a large negative (attractive) force followed by a gradual monotonic decrease in magnitude. This effect is due to the adverse negative pressure gradient which has to develop immediately on either side of the contact point to decelerate the outward transverse motion of the fluid, which has been generated by the cylinder motion before impact. 

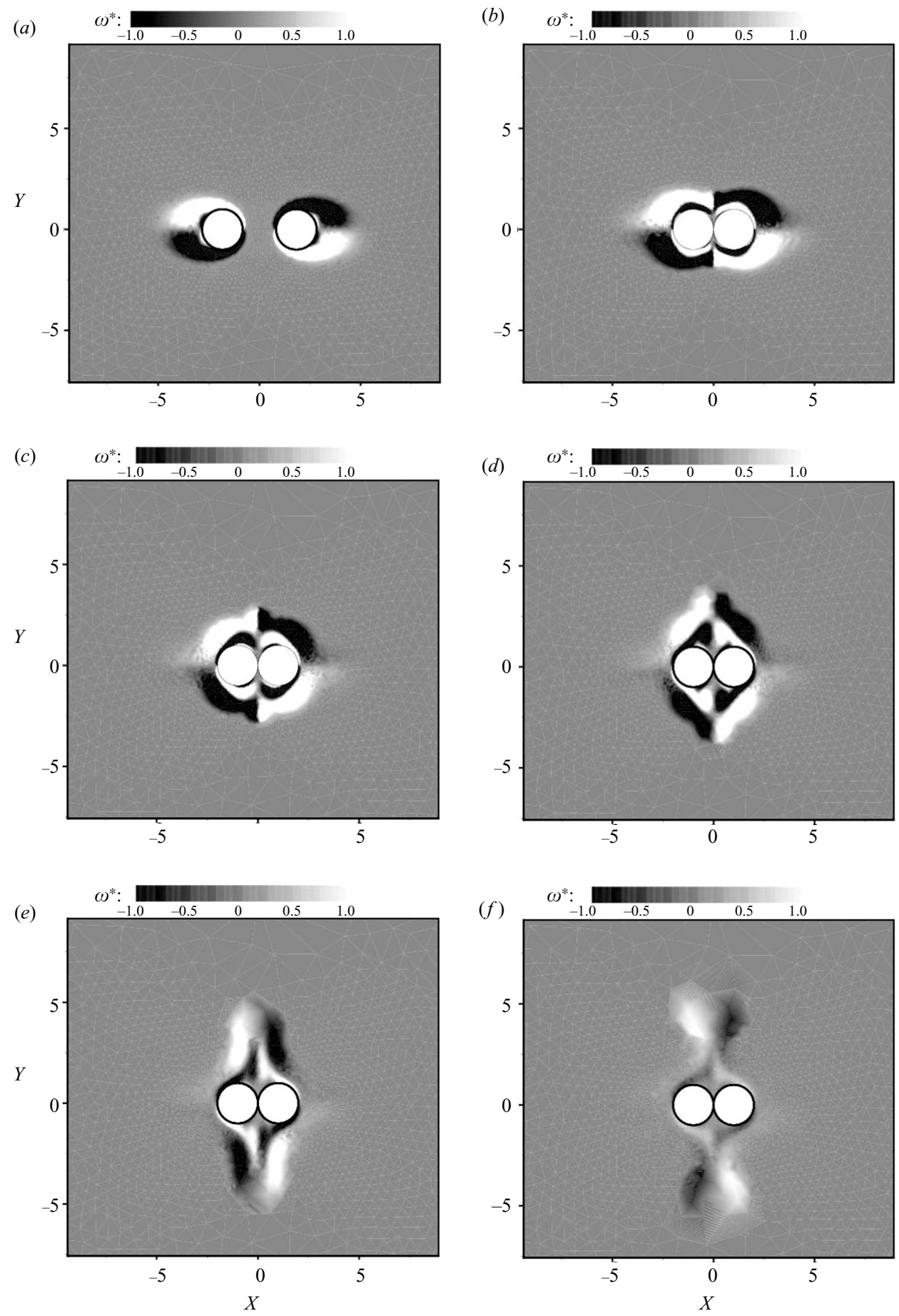

FIGURE 16. Vorticity contour plots for the case of plane impact of two cylinders with zero free stream at successive time steps. 


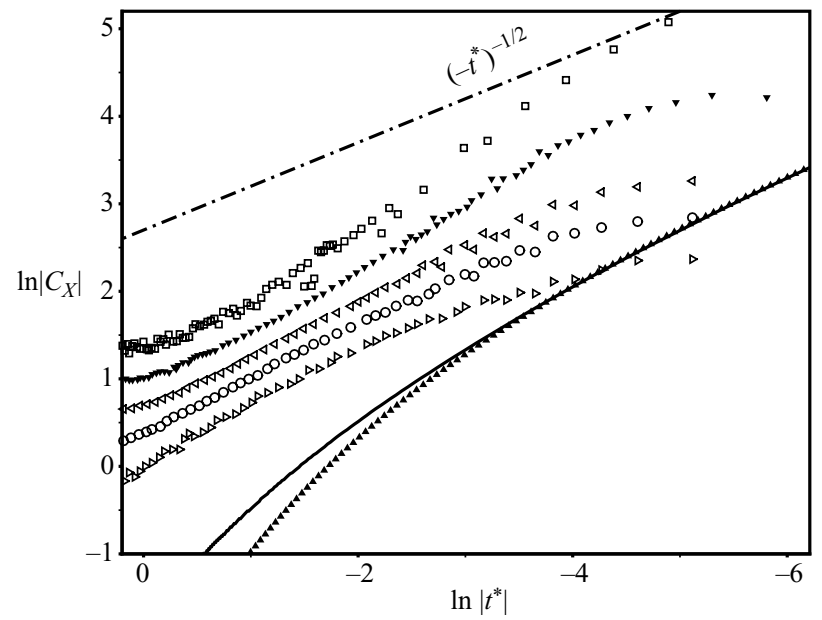

FIGURE 17. Logarithmic plot of $C_{X}$. Symbols as in figure 15.

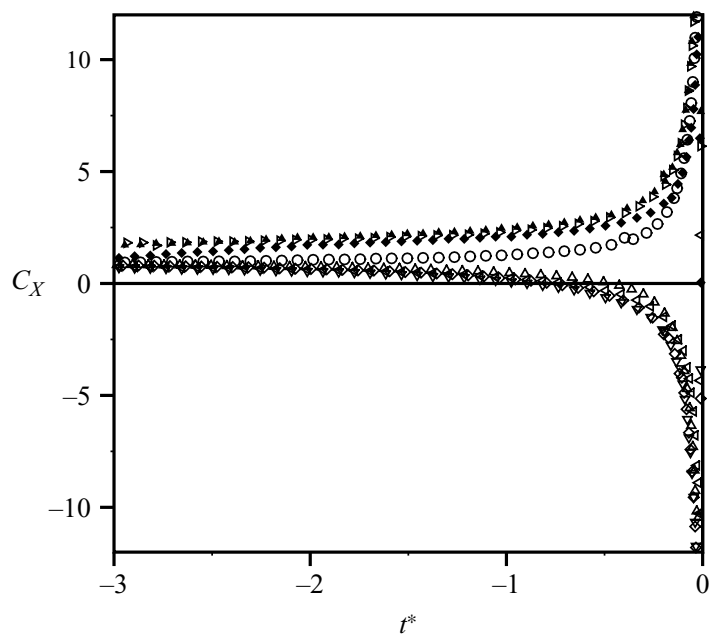

FIGURE 18. Force coefficient $C_{X}$ as cylinders arranged in-line, approach impact in a uniform stream $\left(R e=100, L / D=3\right.$ and $U_{x} / U_{\infty}=1$ except as shown). Upstream cylinder: $\diamond, \triangle$, $\left(U_{x} / U_{\infty}=0.5\right) ; \nabla,(L / D=5) ; \triangleleft,(R e=200)$. Downstream cylinder: $\boldsymbol{\Delta}, O,\left(U_{x} / U_{\infty}=0.5\right)$; $\triangleright,(L / D=5) ; \diamond,(R e=200)$.

\subsection{Cylinder impact in a uniform stream: in-line cylinder arrangement}

In this section the impact of the cylinders is considered in the presence of a free stream. In such cases the pre-existing vortex wakes of the cylinders before they start to interfere become relevant. The flow may no longer be symmetrical, in which case the plane of the shed vortex street in the wake of each cylinder becomes important. As a result many wake-cylinder interactions are possible, and only a few cases have been examined. In the first case with the cylinders arranged in-line with the free stream, the upstream cylinder is held fixed in the uniform flow, and the downstream cylinder, after being held fixed in the flow for the time sufficient enough for both to have started to form asymmetric wakes, moves steadily towards the upstream one until impact. After impact both cylinders are held stationary as earlier. Figure 18 


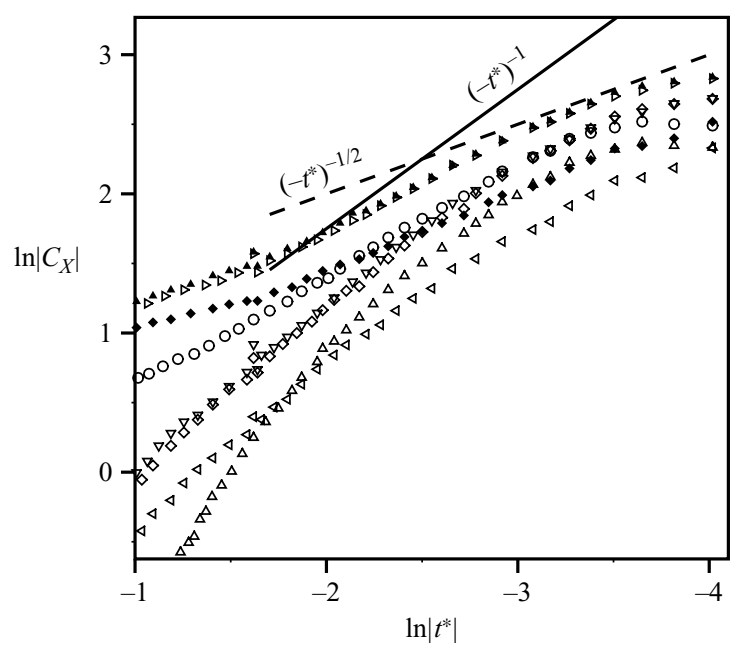

FIGURE 19. Logarithmic plot of the force coefficient $C_{X}$ as cylinders arranged in-line, approach impact in a uniform stream. Symbols as in figure 18.

shows the behaviour of the in-line forces on both cylinders up to impact. These forces are plotted on logarithmic scales in figure 19, showing that they increase at a rate of approximately $t^{*-1}$ for a substantial period before impact. However a final reduction in rate to approximately $t^{*-1 / 2}$ is seen in the final stage up to impact. But this stage for which the separation is less than $5 \%$ of the cylinder diameter may be affected by grid resolution.

In figure 20 vorticity contour plots are shown for the case in which $U_{x} / U_{\infty}=1$, $R e=100$ and an initial distance between the cylinders equal to $L=5 D$. The Reynolds number for this case is based on the velocity of the free stream. The secondary vortices of the downstream-moving cylinder after impact move at right angles to the line joining the centres of the cylinders. They grow to a diameter approximately equal to the cylinder's diameter. A pair of secondary vortices from the downstream cylinder, under the influence of the free stream, envelope these two vortices. At a much later time, $t^{*}=38.3$, the two cylinders start to generate a single Von Kármán vortex street wake. Figure 19 shows the effect of velocity ratio (cylinder velocity/free stream velocity) and Reynolds number for this type of impact. As for the case of plane impact in the absence of a free stream, the initial distance between the cylinders if at least $3 D$ does not significantly affect the final force coefficient. On the other hand $U_{x} / U_{\infty}$ and $R e$ are found to affect the force, with the ratio of the velocity of the cylinder to the free stream having the greater effect. The influence of these parameters is, as expected, greater on the drag coefficient of the moving cylinder than on the fixed cylinder.

\subsection{Cylinder impact in a uniform stream: side-by-side arrangement}

In this case, the cylinders are considered initially fixed in a side-by-side arrangement. After a period during which steady shedding of their wakes is established, both cylinders start to move impulsively towards each other across the stream with equal and opposite velocities until impact, when their motion terminates, and they remain fixed. When vortex wakes are established behind the cylinders, the phase difference 

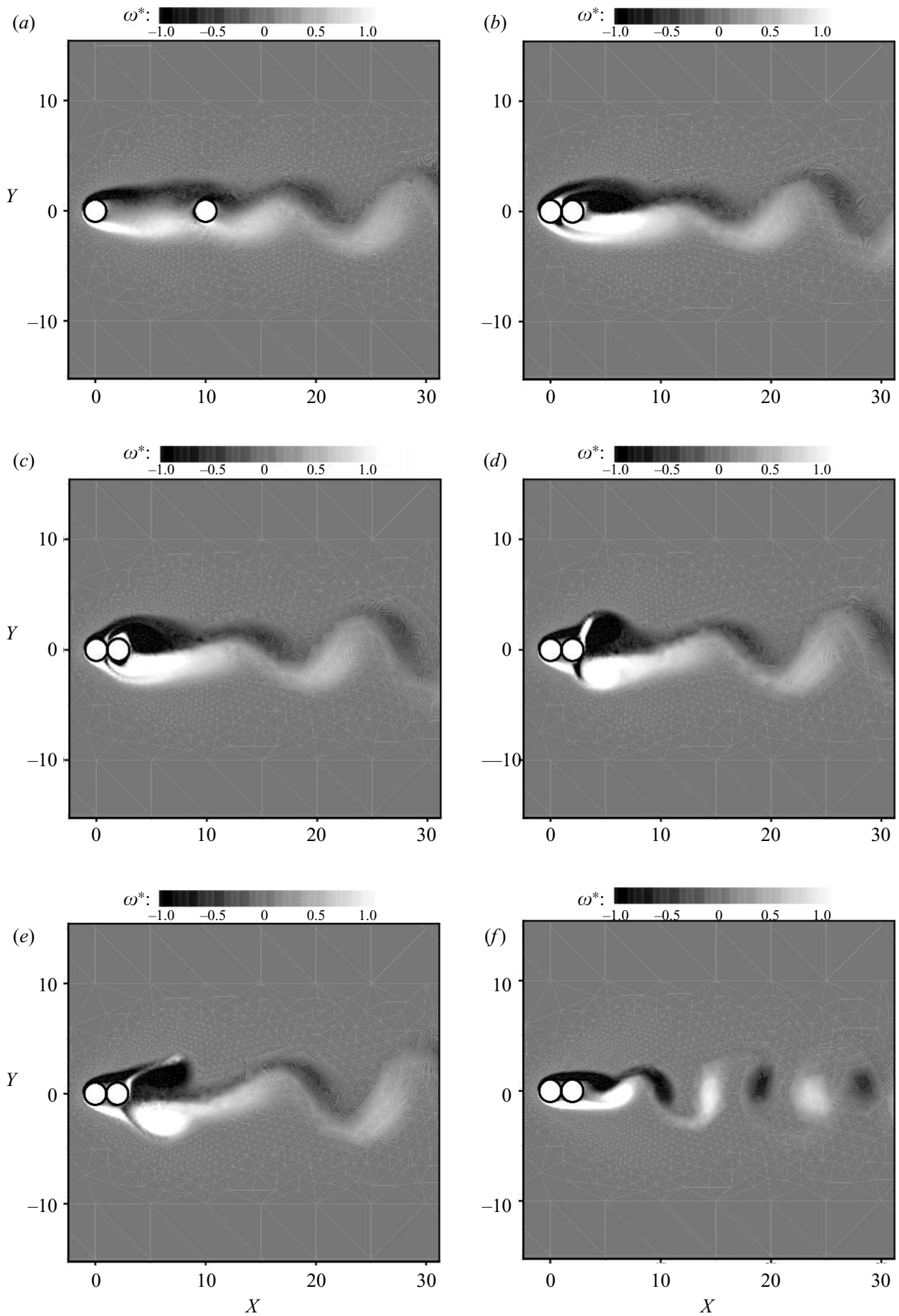

FIGURE 20. Vorticity contour plots at successive time steps for the case of impact of two cylinders arranged in-line, in the presence of a free stream. 


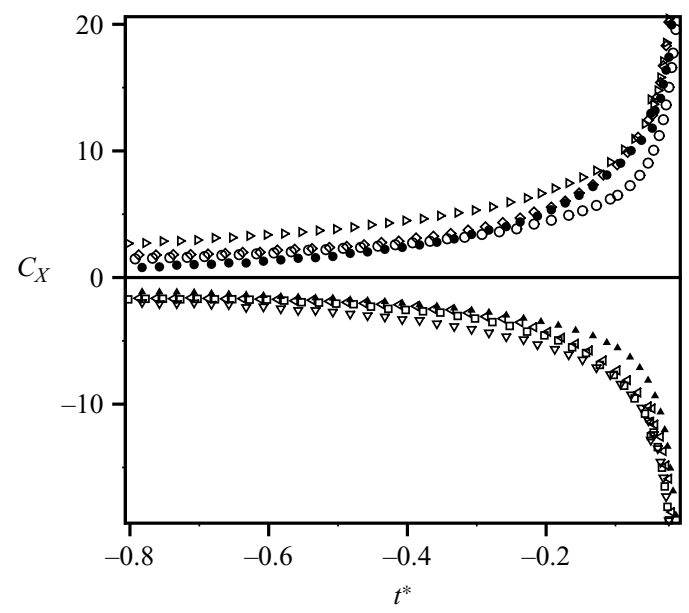

FIgURE 21. The force coefficient $C_{X}$, as the cylinders, aligned side-by-side approach impact, in the presence of a free stream $\left(R e=100, L / D=3\right.$ and $U_{x} / U_{\infty}=1$ except as shown). Lower cylinder: $\square, \boldsymbol{\Delta},\left(U_{x} / U_{\infty}=0.5\right) ; \nabla,(L / D=5) ; \triangleleft,(\operatorname{Re}=200)$. Upper cylinder: $\diamond, \bigcirc$, $\left(U_{x} / U_{\infty}=0.5\right) ; \triangleright,(L / D=5) ; \bullet,(R e=200)$.

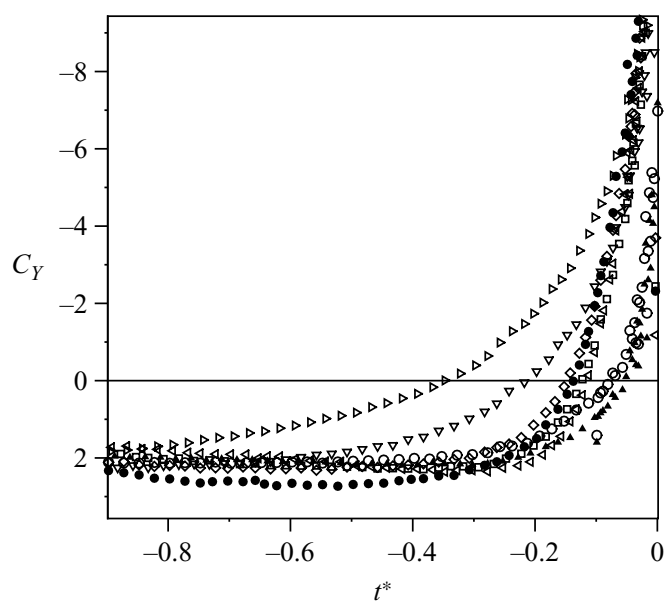

FIGURE 22. The drag force coefficient $C_{Y}$, as the cylinders, aligned side-by-side approach impact, in the presence of a free stream. Symbols as in figure 21.

between the two wakes becomes a relevant parameter for the interaction. However, it was found that for the range of initial distances between the centres of the cylinders tested $(L<5 D)$ the cylinders always tended to shed in-phase (see figure 24) due to mutual, although weak, interference. The inwards motion of the cylinders then caused some changes of phase before impact. After impact, a single-body wake was formed behind the two cylinders. As for the case of in-line arrangement, the influence of varying initial spacing, velocity ratio and Reynolds number were investigated. Figure 22 shows plots of the streamwise force $\left(C_{Y}\right.$ or $\left.C_{D}\right)$ for a range of $L / D, R e$ and $U_{x} / U_{\infty}$ for each cylinder. In the cases considered the initial distance $L=(3 D$ and $5 D)$ between the cylinders does affect the behaviour of the force coefficient close to impact, in contrast to the in-line cases for which the effect of initial distance appeared to be 

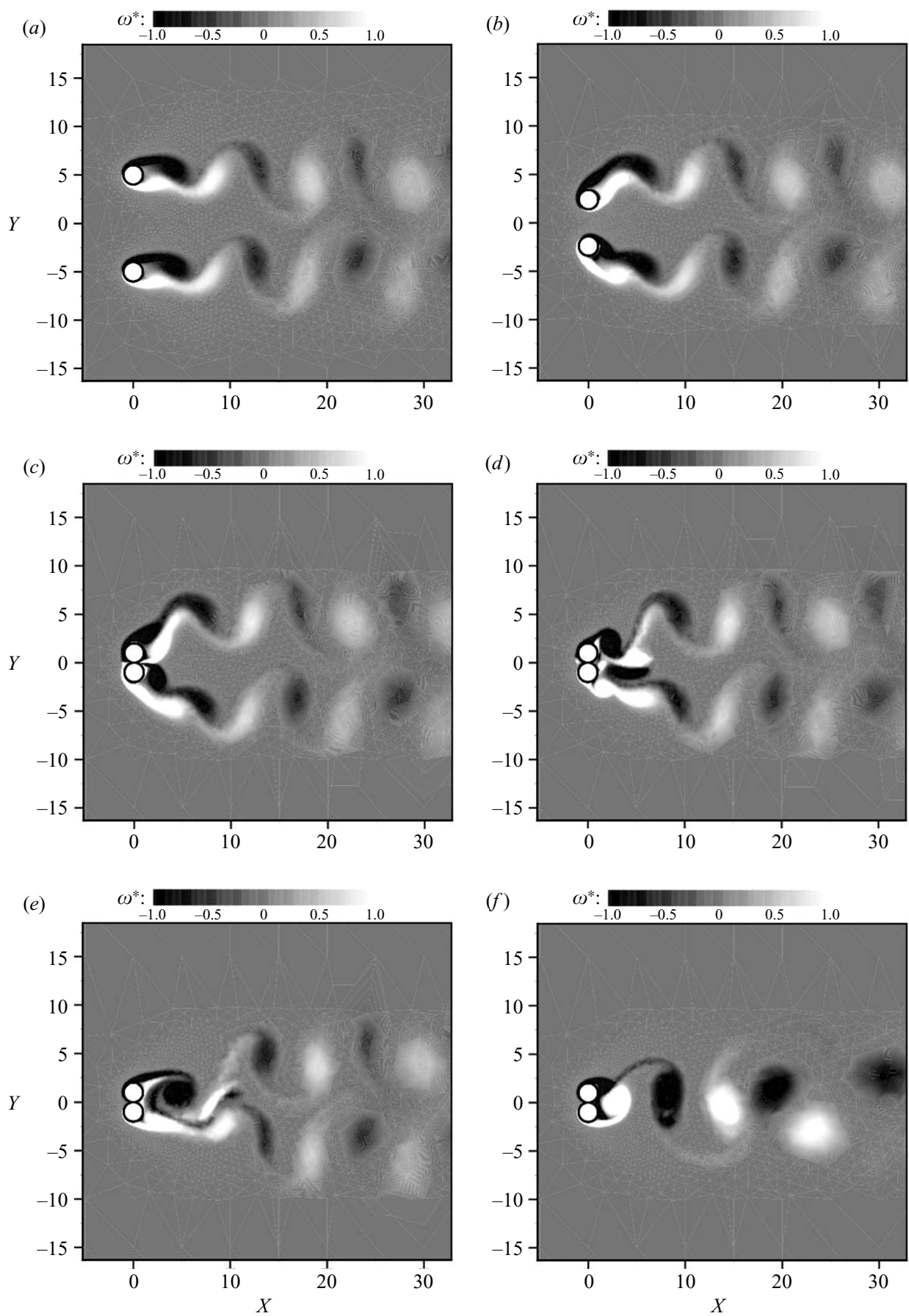

FIGURE 23. Vorticity contour plots at successive time steps for the case of impact of two cylinders arranged side-by-side, in the presence of a free stream.

negligible. Clearly in the side-by-side cases the phase of the vortex shedding has a much stronger influence on the flow through the closing gap than in the tandem cases. The effect on the streamwise force of changing the Reynolds number over the range 
tested is fairly small. It should be noted that the force is always repulsion on both cylinders, although the free stream velocity is as great as or greater than the cylinder velocities in both cases. There are only slight differences in the force amplitudes at the same time due to wake asymmetry.

For all the viscous cases, apart from that of the upstream cylinder in the in-line impact problem, the values of the force coefficient along the line of cylinder centres are quite similar. This suggests that as the gap closes the flow properties in the gap are dominant over other flow field effects such as the wakes of the cylinders and the flow direction. On the upstream cylinder for the in-line arrangement, the in-line force coefficient $C_{X}$ is slightly lower. For all cases, $C_{X}$ appears to increase towards impact at a rate fairly close to $t^{*-1 / 2}$, until mesh resolution effects become significant. In figure 22 the streamwise force coefficient $C_{Y}$ for the side-by-side arrangement is plotted for both cylinders and for different values of the parameters $L / D, U_{x} / U_{\infty}$ and $R e$. At $t^{*} \sim-0.2, C_{Y}$ changes sign for all cases. This unexpected result seems to be due to the narrow gap squeezing fluid out from between the cylinders generating, preferentially because of the incident stream, a downstream jet at impact (see figure 23). This force also similarly appears to increase up to impact approximately as $t^{*-1 / 2}$. Relative to the fluid in these 'side-by-side' cases the cylinders approach one another symmetrically and obliquely. It is reasonable therefore to expect that a component of the very-large-impact repulsion force will be apparent in the streamwise direction, overwhelming the relatively smaller free-stream-induced drag, asymmetry causing this component to be directed forward.

\section{Conclusions}

The flow fields and resulting forces which occur when a pair of circular cylinders move rectilinearly until contact have been numerically simulated assuming twodimensional flow. Cases of both moving cylinders impacting two-dimensionally along their line of centres in otherwise stationary fluid and when immersed in a uniform free stream, have been examined. The first part of the paper treats the fluid as inviscid and computes the flow field by means of conformal transformation and series of image singularities. Results are compared with previously published results and show that when two cylinders move to impact in an otherwise stationary fluid the force of repulsion in the final moments up to impact increases as $t^{*-1 / 2}$, where $t^{*}$ is the dimensionless time to impact, becoming infinite at impact. If an incident free stream normal to the line of centres of the cylinders is also present, the force still varies in the final stages as $t^{*-1 / 2}$ but changes from repulsion to attraction when the velocity of the free stream exceeds the velocity of the cylinders towards their impact point. When the velocities are exactly equal the force remains finite at impact. A narrow gap analysis is developed to confirm these results.

The second part of the paper presents results of viscous flow field computations for the same cases, using a streamfunction-vorticity-based method on an unstructured moving mesh to accommodate the relative cylinder movement. In this case the forces are always found to be repulsion in the final stage of motion up to impact whether a free stream is present or not. The forces increase in magnitude above the inviscid forces as the Reynolds number is reduced. In these cases the viscous-flow-induced force appears to retain the dominant inertia effect as $t^{*} \rightarrow 0$ and increases towards infinite force at impact, with a power of $t^{*}$ of approximately $-1 / 2$. The case of in-line cylinder impact in a free stream is less clear than the other cases in respect of the power of $t^{*}$. 
The moving mesh version of the code VIVIC was developed and validated by Dr T. E. Kendon for this project. The authors gratefully acknowledge this and the financial support of the UK Engineering and Physical Sciences Research Council.

\section{Appendix. Order of integration and differentiation of the complex potential around an expanding closed contour}

A time-dependent expanding closed contour $C$ in the $\zeta$-plane and a complex potential $w(\zeta, t)$ representing a time-dependent flow field in the same plane are considered. Suppose that $w(\zeta, t)$ has $k$ poles within $C$ at the points $a_{i}$, which stay within the contour $C$ as the contour changes with time. Then, the line-integral around $C$ of the time derivative of $w(\zeta, t)$ with respect to time is equal to the time-derivative of the integral of $w(\zeta, t)$ around $C$; i.e. integration in space and differentiation with respect to time may be interchanged even though the integration contour $C$ is a function of time:

$$
\oint_{C} \frac{\partial w(\zeta, t)}{\partial t} \mathrm{~d} \zeta=\frac{\partial}{\partial t} \oint_{C} w(\zeta, t) \mathrm{d} \zeta
$$

In order to derive the last result, $w(\zeta, t)$ is expanded near each of the poles $a_{i}$, in positive and negative powers of $\zeta-a_{i}$ as follows:

$$
w(\zeta, t)=\cdots+A_{i, 2}\left(\zeta-a_{i}\right)^{2}+A_{i, 1}\left(\zeta-a_{i}\right)+A_{i, 0}+\frac{B_{i, 1}}{\zeta-a_{i}}+\frac{B_{i, 2}}{\left(\zeta-a_{i}\right)^{2}}+\cdots
$$

where $A_{i, j}, B_{i, j}$ and $a_{i}$ are time dependent and complex numbers. Therefore,

$$
\begin{aligned}
\frac{\partial w(\zeta, t)}{\partial t}= & \cdots+\frac{\partial A_{i, 2}}{\partial t}\left(\zeta-a_{i}\right)^{2}-2 A_{i, 2} \frac{\partial a_{i}}{\partial t}\left(\zeta-a_{i}\right)+\frac{\partial A_{i, 1}}{\partial t}\left(\zeta-a_{i}\right)-A_{i, 1} \frac{\partial a_{i}}{\partial t} \\
& +\frac{\partial B_{i, 1}}{\partial t} \frac{1}{\zeta-a_{i}}+\frac{\partial a_{i}}{\partial t} \frac{B_{i, 1}}{\left(\zeta-a_{i}\right)^{2}}+\frac{\partial B_{i, 2}}{\partial t} \frac{1}{\left(\zeta-a_{i}\right)^{2}}+\frac{\partial a_{i}}{\partial t} \frac{1}{\left(\zeta-a_{i}\right)^{3}}+\cdots
\end{aligned}
$$

From the last equation, the residue of $\partial w(\zeta, t) / \partial t$ at the $a_{i}$ pole is $\partial B_{i, 1} / \partial t$. By Cauchy's residue theorem the integral of $\partial w(\zeta, t) / \partial t$ around $C$ is given as the following sum:

$$
\oint_{C} \frac{\partial w(\zeta, t)}{\partial t} \mathrm{~d} \zeta=\sum_{i=1}^{k} \frac{\partial B_{i, 1}}{\partial t} .
$$

The integral of $w(\zeta, t)$ around $C$ in now evaluated by directly applying Cauchy's residue theorem. Its value is the sum of the residues inside $C$, and therefore

$$
\frac{\partial}{\partial t} \oint_{C} w(\zeta, t) \mathrm{d} \zeta=\frac{\partial}{\partial t}\left\{\sum_{i=1}^{\infty} B_{i, 1}\right\}
$$

But the right hand sides of (A4) and (A5) are equal, and thus (A1) holds.

\section{REFERENCES}

Bokaian, A. \& Geoola, F. 1984 Wake-induced galloping of two interfering circular cylinders. $J$. Fluid Mech. 146, 383-415.

BRENNER, H. 1961 The slow motion of a sphere through a viscous fluid towards a plane surface. Chem. Engng Sci. 16, 242-251.

Christensen, H. 1962 The oil film in a closing gap. Proc. R. Soc. Lond. A 266, 312-328. 
Crowdy, D. 2006 Analytical solutions for uniform potential flow past multiple cylinders. Europ. J. Mech. B Fluids 25, 459-470.

Dalton, C. \& Helfinstine, R. A. 1971 Potential flow past a group of circular cylinders. J. Basic Engng Trans. ASME, Ser. D 93, 636-642.

Hicks, W. M. 1879 On the motion of two cylinders in a fluid. Quart. J. Math. 16 (2), 113-140, 193-219.

Hicks, W. M. 1880 On the motion of two spheres in a fluid. Philos. Trans. R. Soc. Lond. 171 (2), 455-492.

Koumoutsakos, P., Leonard, A. \& PÉPIn, F. 1994 Boundary conditions for viscous vortex methods. J. Comput. Phys. 113, 52-61.

Landweber, L. \& Shahshahan, A. 1991 Added masses and forces on two bodies approaching central impact in an inviscid fluid. Technical Rep. 346. Iowa Institute of Hydraulic Reserach.

LEE, D. K. 2000 Image singularity system to represent two circular cylinders of different diameter. ASME J. Fluids Engng 122, 715-719.

Milne-Thomson, L. M. 1968 Theoretical Hydrodynamics. Dover.

SAfF, E. B. \& SNider, A. D. 2003 Fundamentals of Complex Analysis with Applications to Angineering and Science. Pearson Education.

Sagatun, S. I., Herfjord, K., Nielsen, F. G. \& Huse E. 1999 Participating mass in colliding risers J. Marine Sci. Technol. 4, 58-67.

Stimson, M. \& Jeffery, G. B. 1926 The motion of two spheres in a viscous fluid. Proc. R. Soc. Lond. 111 (757), 110-116.

VANDIVER, J. K.1993 Dimensionless parameters important to the prediction of vortex-induced vibration of long flexible cylinders in ocean currents. J. Fluids Struct. 7 (5), 423-455.

WANG, Q. X. 2004 Interaction of two circular cylinders in inviscid fluid. Phys. Fluids 16 (12), 4412-4425.

Willden, R. H. J., Graham, J. M. R. 2001 Numerical prediction of VIV on long flexible circular cylinders. J. Fluids Struct. 15, 659-669.

Zdravkovich, M. M. 2003 Flow around Circular Cylinders, Vol. 2: Applications. Oxford Science. 